\title{
Genome-wide identification and characterization of GRAS genes in soybean (Glycine max)
}

Liang Wang, Xianlong Ding, Yingqi Gao and Shouping Yang*

\begin{abstract}
Background: GRAS proteins are crucial transcription factors, which are plant-specific and participate in various plant biological processes. Thanks to the rapid progress of the whole genome sequencing technologies, the GRAS gene families in different plants have been broadly explored and studied. However, comprehensive research on the soybean (Glycine max) GRAS gene family is relatively lagging.

Results: In this study, 117 Glycine max GRAS genes (GmGRAS) were identified. Further phylogenetic analyses showed that the GmGRAS genes could be categorized into nine gene subfamilies: DELLA, HAM, LAS, LISCL, PAT1, SCL3, SCL4/7, SCR and SHR. Gene structure analyses turned out that the GmGRAS genes lacked introns and were relatively conserved. Conserved domains and motif patterns of the GmGRAS members in the same subfamily or clade exhibited similarities. Notably, the expansion of the GMGRAS gene family was driven both by gene tandem and segmental duplication events. Whereas, segmental duplications took the major role in generating new GmGRAS genes. Moreover, the synteny and evolutionary constraints analyses of the GRAS proteins among soybean and distinct species (two monocots and four dicots) provided more detailed evidence for GmGRAS gene evolution. Cis-element analyses indicated that the GMGRAS genes may be responsive to diverse environmental stresses and regulate distinct biological processes. Besides, the expression patterns of the GMGRAS genes were varied in various tissues, during saline and dehydration stresses and during seed germination processes.
\end{abstract}

Conclusions: We conducted a systematic investigation of the GRAS genes in soybean, which may be valuable in paving the way for future GMGRAS gene studies and soybean breeding.

Keywords: Soybean, GRAS, Genome-wide, Evolutionary analyses, Expression patterns, Saline and dehydration stresses, Seed germination

\footnotetext{
* Correspondence: spyung@126.com

Soybean Research Institute, National Center for Soybean Improvement, Key

Laboratory of Biology and Genetic Improvement of Soybean (General,

Ministry of Agriculture), State Key Laboratory of Crop Genetics and

Germplasm Enhancement, Jiangsu Collaborative Innovation Center for Modern Crop Production, College of Agriculture, Nanjing Agricultural

University, Nanjing 210095, China
}

(c) The Author(s). 2020 Open Access This article is licensed under a Creative Commons Attribution 4.0 International License, which permits use, sharing, adaptation, distribution and reproduction in any medium or format, as long as you give appropriate credit to the original author(s) and the source, provide a link to the Creative Commons licence, and indicate if changes were made. The images or other third party material in this article are included in the article's Creative Commons licence, unless indicated otherwise in a credit line to the material. If material is not included in the article's Creative Commons licence and your intended use is not permitted by statutory regulation or exceeds the permitted use, you will need to obtain permission directly from the copyright holder. To view a copy of this licence, visit http://creativecommons.org/licenses/by/4.0/. The Creative Commons Public Domain Dedication waiver (http://creativecommons.org/publicdomain/zero/1.0/) applies to the data made available in this article, unless otherwise stated in a credit line to the data. 


\section{Background}

The GRAS transcription factors (TFs) are plant-specific regulating proteins that have been widely studied in the past decade [1-4]. The name of GRAS proteins was derived from the three first identified members of the gene family: gibberellic acid insensitive (GAI), repressor of GA1-3 mutant (RGA), and scarecrow (SCR) [5]. In general, the GRAS protein sequences consisted of 400-770 amino acid residues, which exhibited highly conserved C-terminal regions and variable $\mathrm{N}$-terminals $[6,7]$. Commonly, the GRAS domains were determined by the conserved carboxyl-terminal regions, and could be divided into five motifs: leucine-rich region I (LHRI), VHII D, leucine-rich region II (LHRII), PFYRE, and SAW. Notably, these five motifs played important roles in the interactions between GRAS with other proteins [8]. According to the early research, LHRI and LHRII were crucial for the homologous dimerization of GRAS proteins. The VHIID motif was the core component of the GRAS protein, which contained a very conserved P-N-H-D-Q$\mathrm{L}$ unit and ended with L-R-I-T-G. Three pairs of conserved protein sequence characters, P, FY, and RE, could be recognized and assembled into the PFYRE motif, which might be correlated to phosphorylation. And the SAW motif consisted of three conserved amino acid residues: R-E, W-G, and W-W $[4,6,9]$. By contrast, the fickle N-terminus of GRAS proteins could be folded and modified into specific molecular binding structures. Based on these, the GRAS proteins broadly participated in many critical processes such as signal transductions, root radial elongations, axillary shoot meristem formations and stress responses in plants [10-13]. Previously, the GRAS gene family in Arabidopsis thaliana was separated into eight subfamilies, including DELLA, SCL3, LAS, SCR, HAM, SHR, LISCL and PAT1 [14]. The DELLA subfamily contained the GAI, RGA and RGL genes, and was reported as the main repressors of gibberellin signal transduction [15]. Importantly, the SCL3 proteins were validated as the switches of mediating the elongation of the root [16]. Moreover, the SCL3 proteins could cooperate with the DELLA proteins and adjusted gibberellin feedback via IDD proteins [17]. Besides, SHR and SCR proteins tended to form the SCR/SHR complex, which was determined to be associated with root radial patterning $[18,19]$. LAS proteins were reported tightly linked to the lateral shooting formation during the vegetative growth stages of Arabidopsis [11]. Furthermore, the overexpression of VaPAT1 (a GRAS gene of Vitis amurensis) improved the abiotic stress tolerance in the transgenic Arabidopsis [20]. Another study turned out that AtSCL13 (a member of the PAT1 subfamily in Arabidopsis thaliana) involved in phytochrome A (phyA) signal transduction and played a major role in hypocotyl elongation [21]. In Medicago truncatula, the
HAM subfamily gene MtNSP2 together with the MtNSP1 (the SHR subfamily gene), formed a DNA binding complex to induce gene expression during nodulation signaling [22]. In Petunia, the PhHAM genes acted on adjacent tissues in noncellular autonomous ways and maintained the activities of the apical meristem [23]. With the rapid development of sequencing technologies, several new subfamilies, for instance, DLT, SCL4/7, Os19, Os4 and PT20, gradually enriched the former GRAS gene subfamilies in diverse plants [24]. To date, there are over 30 mono- and dicotyledonous plants, such as rice, maize, Arabidopsis, cotton, Malus domestica and castor beans have been carried out genome-wide GRAS gene family identifications and analyses [3, 8, 19, 25, 26].

Soybean (Glycine $\max$ L.) is one of the major crops abundant in high-quality protein and oil, which also contains various nutrients such as lecithins and isoflavones [27]. Many soybean transcription factor families like WKRY [28], MYB [29], NAC [30], HD-Zip [31], ARF [32] and MADS [33] have been investigated and studied. However, comprehensive studies on the Glycine max GRAS gene (GmGRAS) family are relatively lagging. Owing to the importance of the GRAS genes in plant developmental and physiological courses, it is imperative to conduct relevant explorations and analyses to fix the gap. In this study, we systematically identified 117 GmGRAS gene members from the soybean genome. First, we investigated the phylogenetic relations, gene structures, motif compositions, chromosomal locations and gene duplication events of the identified GmGRAS members. Next, we carried out the evolutionary analyses on the GRAS members among soybean and four dicotyledons (Arabidopsis thaliana, Glycine soja, Vigna unguiculata and Solanum lycopersicum) as well as two monocotyledons (Oryza sativa and Sorghum bicolor). Moreover, we analyzed cis-elements in promoter regions of the GmGRAS genes. Besides, we explored the expression patterns of the GmGRAS genes in different tissues, during saline and dehydration stresses and during seed germination processes. In particular, due to the importance of seed germination in soybean production, 18 representative soybean GRAS genes were further selected and carried out the quantitative RT-PCR analyses. Collectively, the current research provided insights for the future functional study of GmGRAS genes and may be valuable for soybean breeding.

\section{Results}

\section{Identification of GmGRAS genes in soybean}

Totally 117 GmGRAS genes were identified from the soybean Wm82.a2.v1 genome on Phytozome (https://phytozome.jgi.doe.gov/pz/portal.html\#). Among them, 116 genes were mapped on the 20 different soybean chromosomes and one gene (Glyma.U013800.1.Wm82.a2.v1) was 
located on unattributed scaffold_21 of the soybean genome, which was renamed as GmGRAS117. According to the chromosome names and chromosomal locations, the rest 116 GmGRAS genes were renamed from GmGRAS1 to GmGRAS116, respectively (Additional file 1: Table S1).

The basic characteristics of GmGRAS family members were listed in Table S1 (Additional file 1), including the open reading frame (ORF) length, the protein size, the protein molecular weight $(\mathrm{MW})$, isoelectric point (pI), the predicted subcellular localization, the putative conserved domain, homologs in other species. As is shown in Table S1 (Additional file 1), GmGRAS55 was the smallest protein with 169 amino acids (aa), whereas the largest one was GmGRAS111 (843 aa). The MW of the proteins spanned from $18,975.84$ to $91,543.91 \mathrm{Da}$, and the pI ranged from 4.76 (GmGRAS33) to 9.21 (GmGRAS55). The predicted subcellular localization results showed that 74 GmGRAS proteins were located in the nuclear region, 29 in the cytoplasm, ten in the plasma membrane, two in the extracellular region, one in the chloroplast, and one in the mitochondria. And the coding sequences and the protein sequences of the identified GmGRAS gene members were listed in Table S2 (Additional file 2).

\section{Phylogenetic analyses and classifications of GmGRAS} gene members

To classify the phylogenetic relationships of soybean GRAS proteins, we constructed a phylogenetic tree based on the identified 117 GmGRAS in this study and 32 reported Arabidopsis GRAS proteins from TAIR (https://www.arabidopsis.org/index.jsp) (Additional file 3: Table S3). The phylogenetic analyses showed that the 116 GmGRAS gene members were divided into nine subfamilies: DELLA, HAM, LAS, LISCL, PAT1, SCL3, SCL4/7, SCR, SHR. Comparably, GmGRAS55 was relatively independent, which did not belong to any GRAS gene subfamilies (Fig. 1). As is shown in Fig. 1 and Table S1 (Additional file 1), the PAT1 subfamily herein contained 23 members and was the largest GmGRAS gene subfamily in this study. The LISCL subfamily was one gene member less than the PAT1 subfamily. Coincidentally, both the HAM and SCL3 subfamilies included 15 members. Besides, there were 14, 13, 6, 6 and 2 GmGRAS gene members in the DELLA, SHR, SCR, SCL4/7 and LAS subfamilies, respectively.

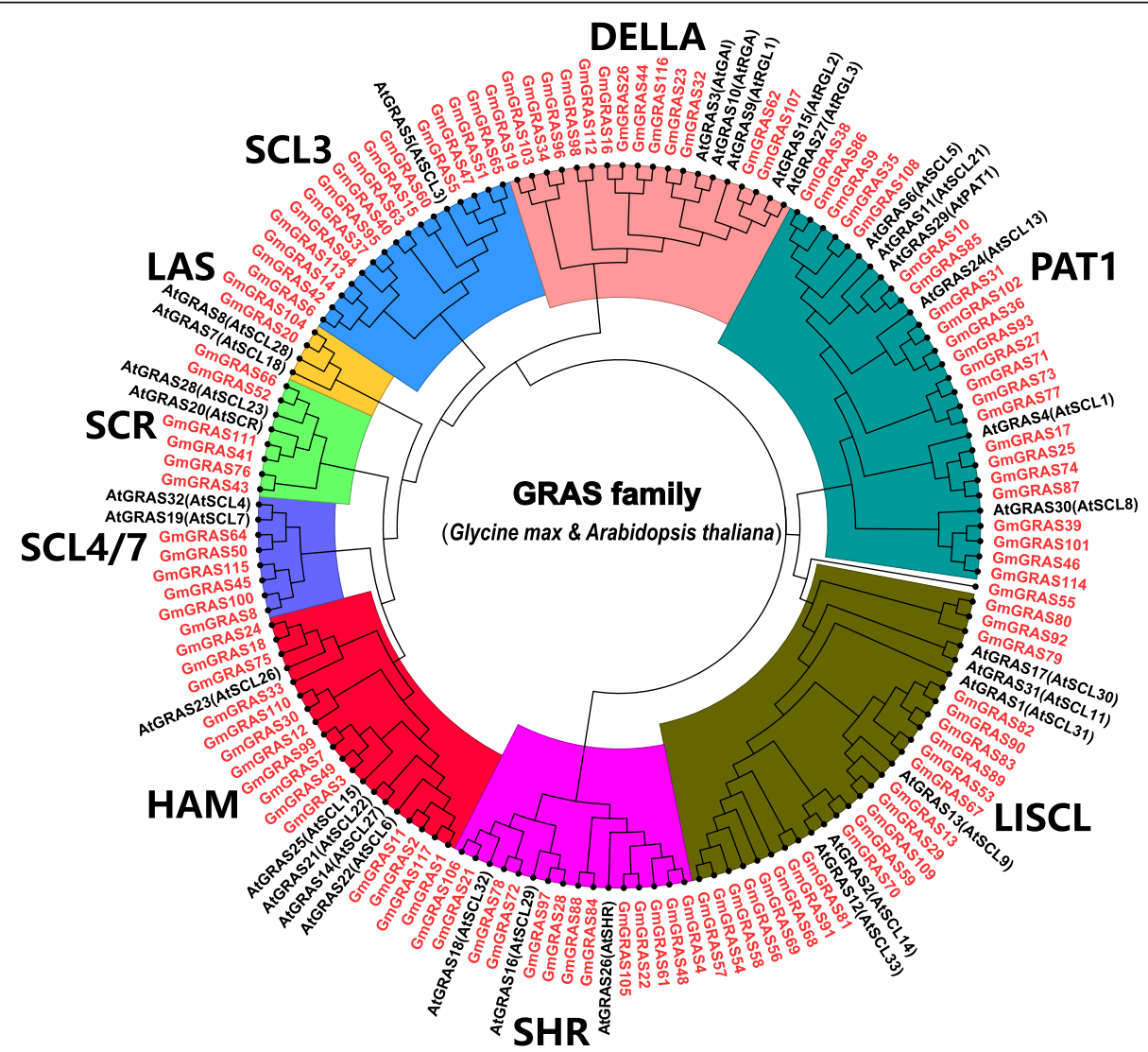

Fig. 1 Unrooted phylogenetic tree of GRAS proteins in soybean and Arabidopsis. The GRAS protein sequences of the two species were aligned by MEGA 7.0 with the MUSCLE method, and the tree was built with the neighbor-joining (NJ) method. The tree was further categorized into nine distinct subfamilies in different colors. All the GmGRAS proteins have been emphasized in red 


\section{Gene structures and motif patterns of GmGRAS gene} members

By screening the corresponding genomic DNA sequences and the annotation files, the exon-intron patterns of the identified GmGRAS genes were obtained. As is shown in Fig. 2, the GmGRAS genes displayed one to seven exons (91 with one exon, 14 with two exons, five with three exons, five with four exons, one with five exons, and one with seven exons) and lacked introns.
For the protein conserved domains, all the 117 GmGRAS members possessed at least one GRAS or GRAS superfamily domain. Members in the same subfamily or clade have similar gene structures and protein conserved domains. For instance, GmGRAS16, GmGRAS23, GmGRAS26, GmGRAS32, GmGRAS44 and GmGRAS62 belonged to the DELLA subfamily, and each of them contained a DELLA protein domain with one exon and no intron (Fig. 2).

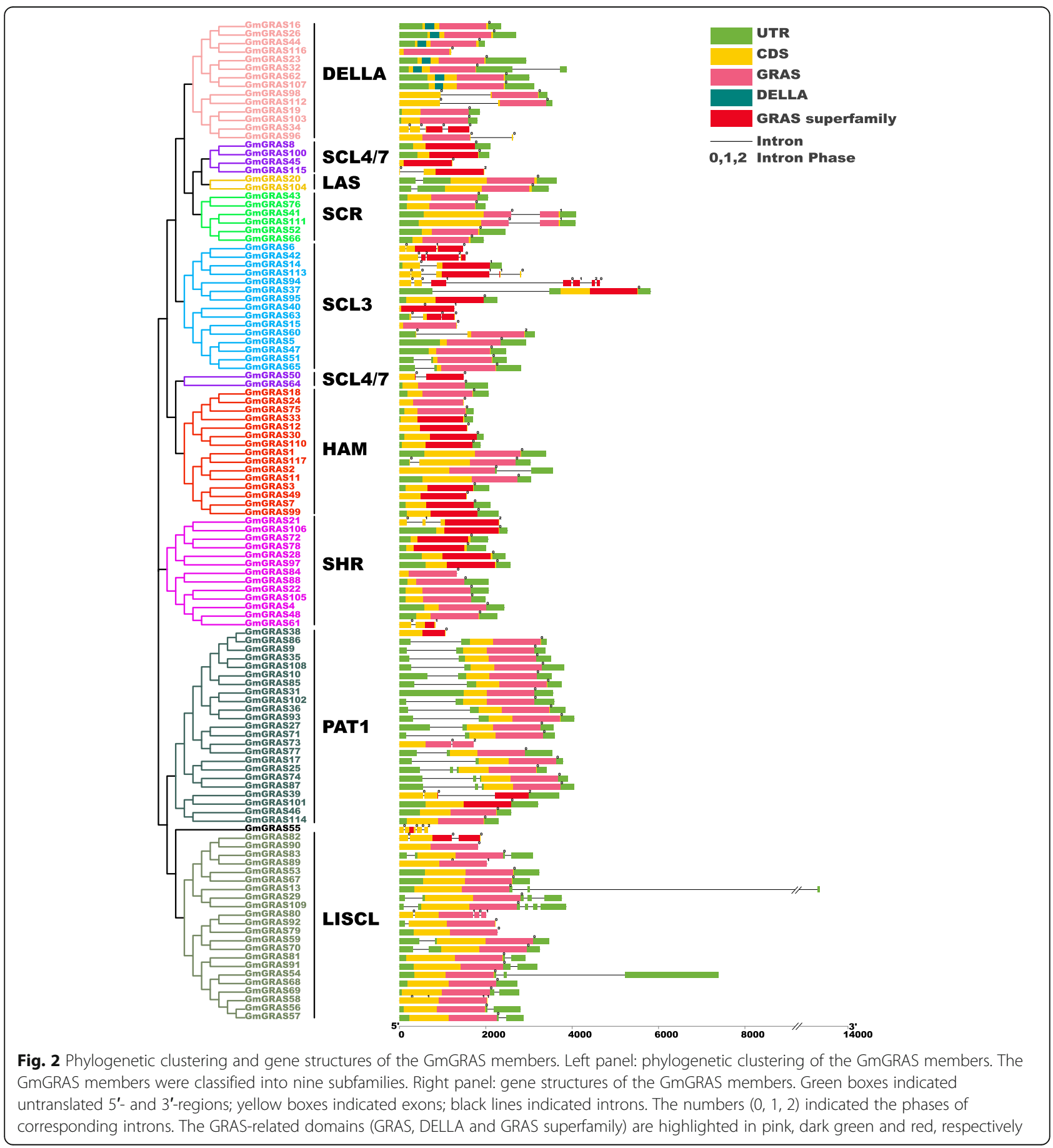


To further demonstrate the structures of the GmGRAS proteins, a schematic was built based on the MEME-motif scanning result. As is shown in Fig. 3b, 20 diverse MEMEmotifs (named Motif 1-20) were displayed. Moreover, the details of these motifs were presented in Table S4 (Additional file 4) and the Seq Logos of the 20 MEME-motifs were exhibited in Fig. S1 (Additional file 5). Referring to the classifications of Quan et al. in Juglans regia, the MEME-motifs were further assessed and categorized into the five GRAS specific C-terminal motifs: LRHI, VHIID, LRHII, PFYRE and SAW [34]. As a result, Motifs 7 and 10 were classified into the LRHI motif; Motifs 1 and 11 belonged to the VHIID motif; Motifs 6 and 9 were associated with the LRHII motif; Motifs 3, 8 and 12 were included by the PFYRE motif; and Motifs 2, 4, 14 and 16 were in the SAW motif (Fig. 3b and Fig. 3c). Besides, Motifs 5, 15 and 18 were located between the LRHI and VHII $\mathrm{D}$ motifs. It is worth noting that the MEME-motifs in the

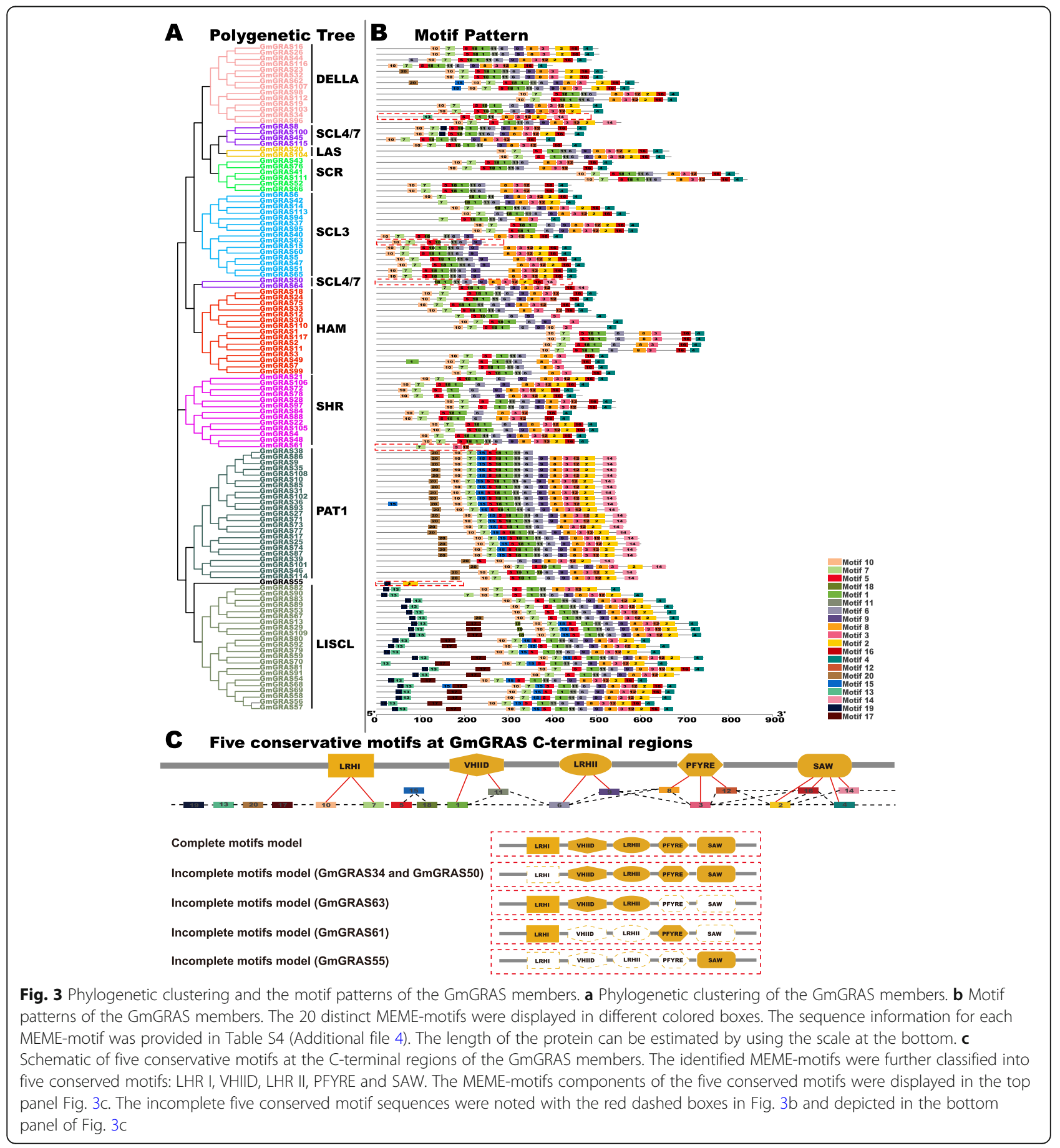


five GRAS specific C-terminal motifs were not fixed. Sometimes, merely one MEME-motif existed in the Cterminal conserved motifs. However, some C-terminal conserved motifs were corresponding to two or three MEME-motifs. Interestingly, some soybean GRAS subfamilies contain unique MEME-motifs. For instance, the Motifs 13, 17 and 19 were only found in the LISCL subfamily. As is shown in Fig. 3b and Fig. 3c, most GmGRAS proteins contain the complete components of the five conservative motifs at C-terminals, however, with the exceptions that GmGRAS34, GmGRAS50, GmGRAS55, GmGRAS63 and GmGRAS61 lacked one to four subunits of the five conserved motifs (denoted with the red dotted boxes). Overall, the MEME-motifs in the specific GmGRAS gene subfamily or clade exhibited similar components and displayed orders.

\section{Chromosomal distributions, synteny and evolutionary analyses of GmGRAS gene members}

The GmGRAS gene chromosomal positions were depicted based on the gene physical location information of the soybean genome (Fig. 4). Importantly, the gene density of each chromosome or scaffold was also evaluated by setting the genetic interval as $300-\mathrm{kb}$ in Table $\mathrm{S} 5$ (Additional file 6) and was further illustrated by gradient colors from blue (low gene density) to red (high gene density) in Fig. 4. The blank regions on chromosome or scaffold indicated that the genetic regions lacked gene distribution information. As is shown in Fig. 4, the 117 GmGRAS genes were unevenly distributed on the 20 soybean chromosomes (Chr01 - Chr20) and scaffold_21. And most identified GmGRAS genes tended to locate in the high gene density regions. Notably, Chr11 contained

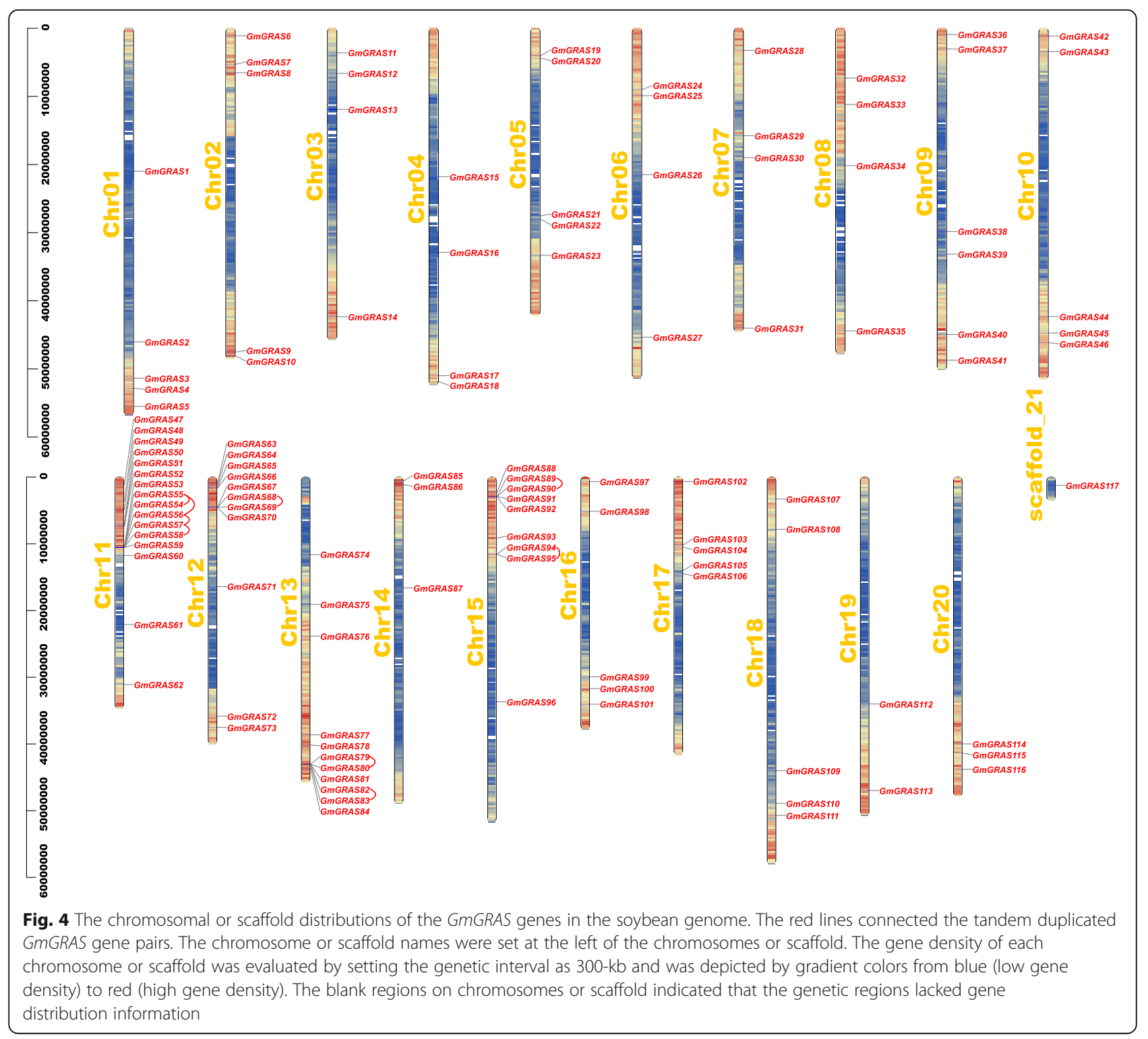


the most GmGRAS genes and 16 genes were located on this chromosome. Some chromosomes (e.g. Chr12, Chr13 and Chr15) have considerable GmGRAS gene members, whereas some (e.g. Chr19) have few, and there is only one GmGRAS gene on scaffold_21. Similar to the previous studies on GRAS genes in other species, no obvious correlation was found between the chromosome length and the number of GmGRAS genes [4, 7].

Early research demonstrated that gene duplications were essential for the occurrences of new gene functions and the expansions of the gene families [35]. Hence, we further explored the duplication events of the identified 117 GmGRAS genes (Fig. 4 and Fig. 5). In a previous study, Holub defined a tandem duplication event as a $200 \mathrm{~kb}$ (kilobase) intergenic region containing multiple (two or more) gene family members [36]. Comparably, segmental duplications frequently happened in plants because most plants are diploidized polyploids and retain numerous duplicated chromosomal blocks within their genome [37, 38]. And segmental duplications multiple genes through polyploidy followed by chromosome rearrangements [39]. Importantly, both segmental and tandem duplications were considered to be two representative main causes of gene family expansion in plants $[37,38]$. In this study, fifteen GmGRAS genes were found in nine tandem duplication events (GmGRAS54/GmGRAS55, GmGRAS55/GmGRAS56, GmGRAS56/GmGRAS57, GmGRAS57/GmGRAS58, GmGRAS68/GmGRAS69, GmGRAS79/GmGRAS80, GmGRAS82/GmGRAS83, GmGRAS89/GmGRAS90 and GmGRAS94/GmGRAS95) distributed on four soybean chromosomes (Chr11, Chr12, Chr13 and Chr15), which turned out these regions may be the hotspots for GmGRAS gene distributions (Additional file 7: Table S6). Notably, most tandem duplication events happened in the LISCL subfamily, except for GmGRAS94/ GmGRAS95, which occurred in the SCL3 subfamily. Furthermore, 104 segmental duplication events associate with 107 GmGRAS genes were also detected (Fig. 5 and Additional file 7: Table S6). In summary, most GmGRAS genes possibly originated from the gene duplications, and the segmental duplication events may play a pivotal role in generating new GmGRAS genes.

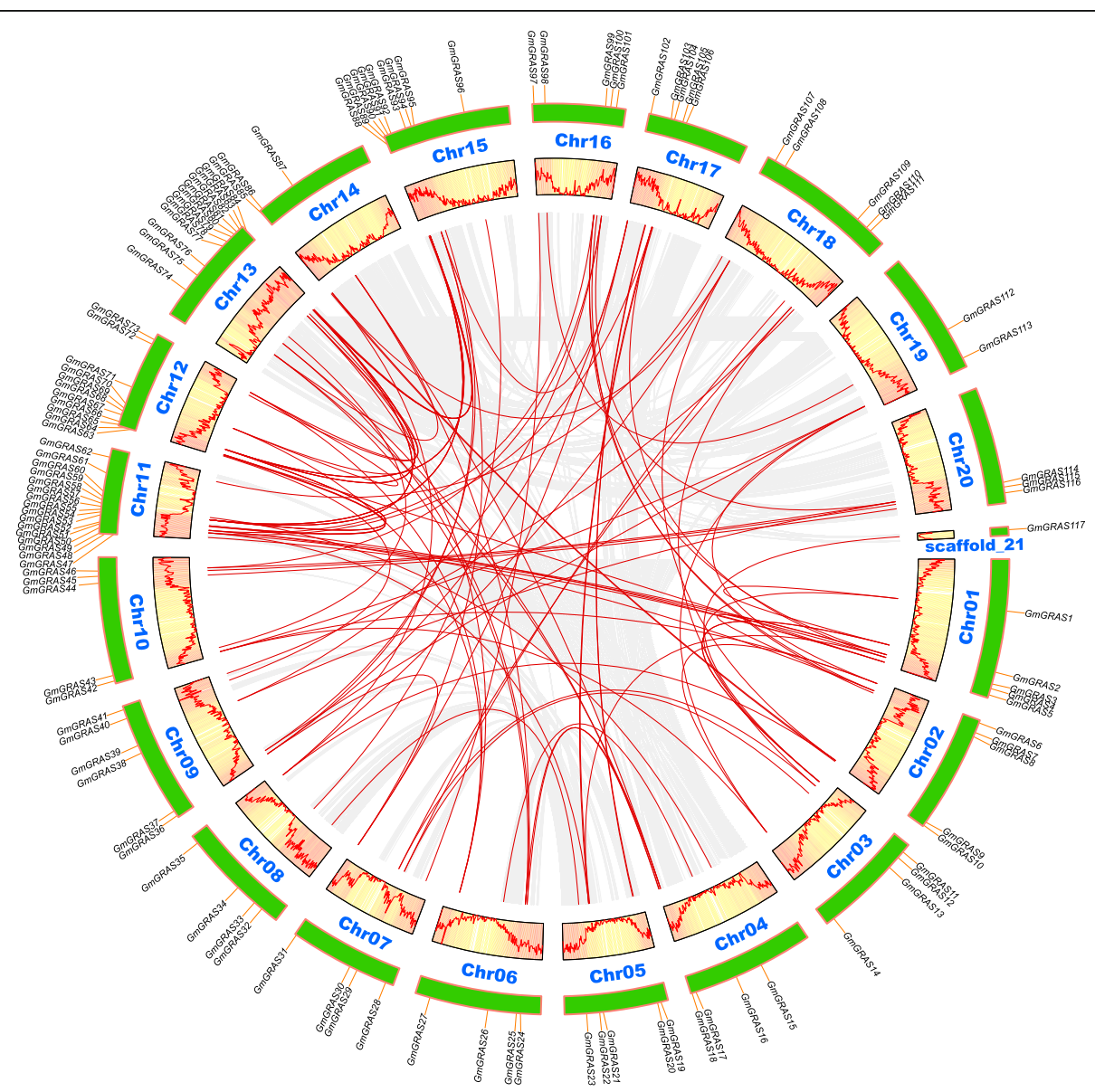

Fig. 5 Inter-chromosomal relations of the GMGRAS genes in the soybean genome. All the syntenic blocks in the soybean genome were depicted by the gray lines, and the red lines linked the duplicated GRAS gene pairs. The gene density of 300-kb hereditary interval on each chromosome or scaffold was depicted by the heatmap and the wave graph 
To explore the evolutionary clues for the soybean GRAS gene family, we constructed six comparative syntenic graphs to display the synteny of GRAS gene members between soybean and six representative species (Fig. 6). The six representative species contained four dicots (Arabidopsis thaliana, Glycine soja, Vigna unguiculata and Solanum lycopersicum) and two monocots (Oryza sativa and Sorghum bicolor). Totally 109 GmGRAS gene members showed syntenic relationships with those in Glycine soja (101), Vigna unguiculata (49), Solanum lycopersicum (31), Arabidopsis thaliana (22), Sorghum bicolor (21) and Oryza sativa (9) (Additional file 8: Table S7). And the numbers of GmGRAS orthologous genes in Glycine soja, Vigna unguiculata, Solanum lycopersicum, Arabidopsis thaliana, Sorghum bicolor and Oryza sativa were 289, 162, 104, 64,
57 and 29, respectively. Overall, the GmGRAS genes consisted of more syntenic gene pairs with dicots compared to those in monocots. Furthermore, as the ancestor of Glycine max (soybean), Glycine soja exhibited superior synteny with soybean than the other five species. Importantly, as is shown in the interactive Venn diagram of GRAS genes throughout the different species (Fig. 7a), 19 GmGRAS genes had syntenic GRAS gene pairs in all the six species. And the 19 GmGRAS genes were highlighted in bold in Table S8 (Additional file 9). The syntenic gene pairs between soybean and other species may be valuable to illuminate the evolutions of GRAS genes.

In this study, the $\mathrm{Ka} / \mathrm{Ks}$ (non-synonymous substitution/synonymous substitution) ratios of the GmGRAS orthologous gene pairs in the six species were calculated

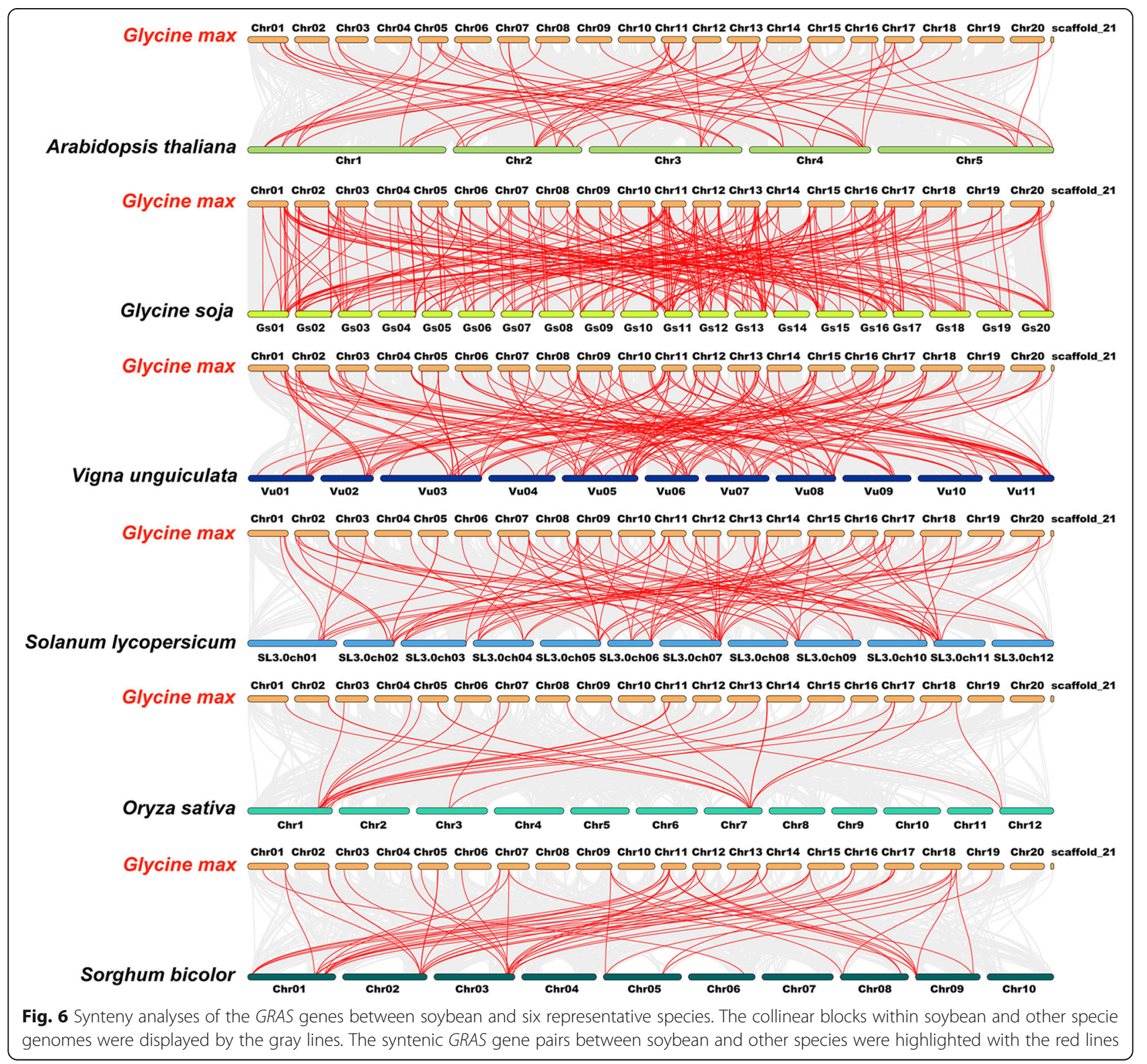



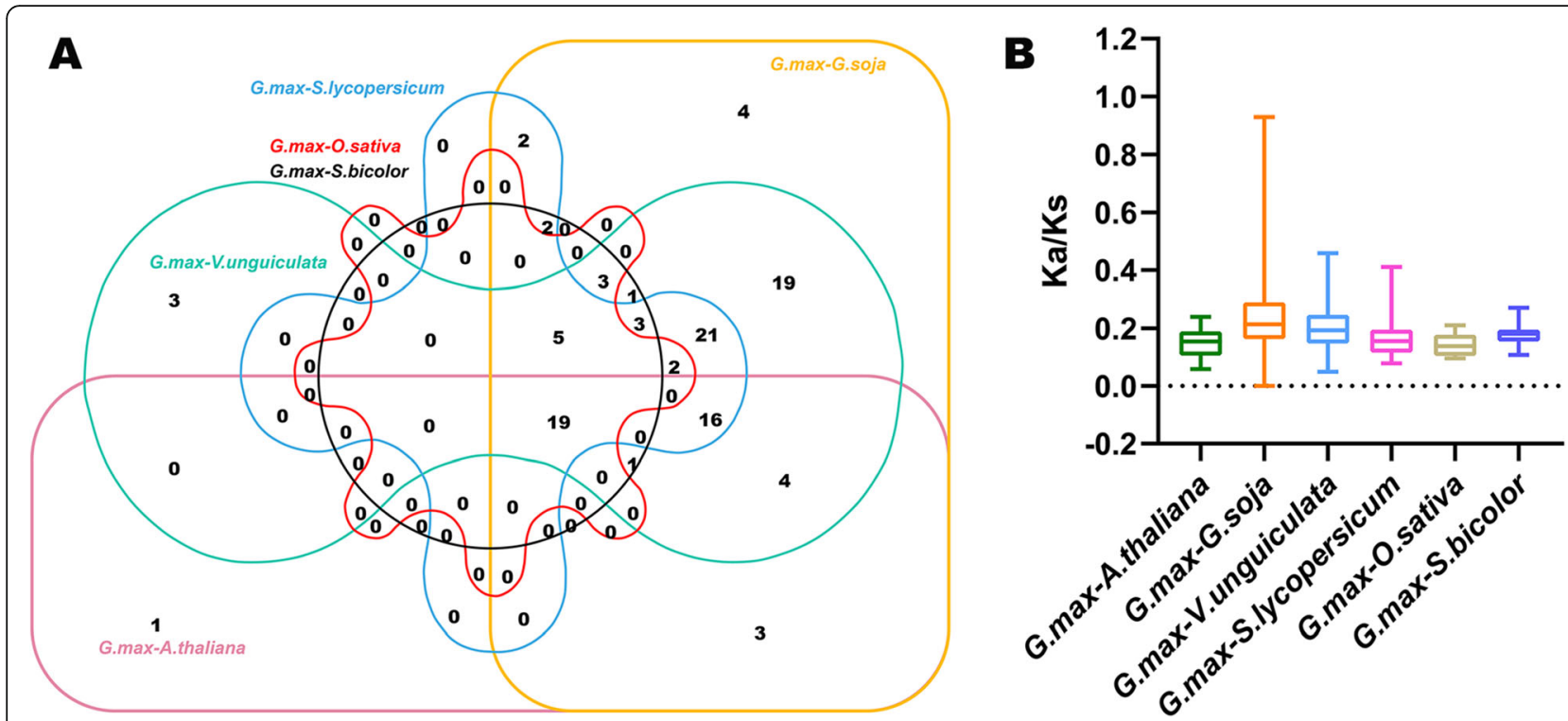

Fig. 7 Non-redundant syntenic GMGRAS genes throughout diverse species and evolutionary analyses of the GRAS gene families. a The Venn diagram of syntenic GRAS genes throughout diverse species. $\mathbf{b}$ The ratio of nonsynonymous to synonymous substitutions (Ka/Ks) of GRAS genes in soybean and other six species. The species' names with the prefixes 'G. max', 'A. thaliana', 'G. soja', 'V. unguiculata', 'S. Iycopersicum', 'O. sativa' and 'S. bicolor' indicated Glycine max, Arabidopsis thaliana, Glycine soja, Vigna unguiculate, Solanum lycopersicum, Oryza sativa and Sorghum bicolor, respectively

to evaluate the evolutionary constraints acting on soybean GRAS gene family (Additional file 8: Table S7). As is shown in Fig. 7b, all GmGRAS orthologous gene pairs displayed $\mathrm{Ka} / \mathrm{Ks}<1$. Hence, we speculated that the soybean GRAS gene family might go through strong purifying selective pressures during the evolution [35].

\section{Cis-element analyses of soybean GmGRAS genes}

The cis-elements play an essential role in transcriptional regulation of the gene expression [40]. In this study, the 2000-bp upstream sequences of the identified GmGRAS genes were extracted from the soybean genome, and the cis-element analysis was carried out by using PlantCARE (http://bioinformatics.psb.ugent.be/webtools/plantcare/ $\mathrm{html} /$ ) (Additional file 10: Table S9). As is displayed in Fig. 8 and Table S9 (Additional file 10), 16 types of ciselements were acquired in 2000-bp promoter regions of the GmGRAS genes. Notably, the cis-elements like light responsive, auxin responsive, gibberellin responsive, abscisic acid responsive, MeJA responsive, defense and stress responsive, drought inducibility and anaerobic induction were broadly distributed, which indicated the GmGRAS gene diversely response to different abiotic stresses and regulate various biological processes.

\section{Expression profiles of GmGRAS genes in various tissues and gene expression correlation analyses}

To investigate the expression profiles of the GmGRAS gene members during soybean different developmental stages in diverse tissues, we extracted and analyzed the transcript levels of the GmGRAS genes in young leaf, flower, one $\mathrm{cm}$ (centimeter) pod, pod shell $10 \mathrm{DAF}$ (days after flowering), pod shell 14 DAF, seed 10 DAF, seed 14 DAF, seed 21 DAF, seed 25 DAF, seed 28 DAF, seed 35 DAF, seed 42 DAF, root and nodule [41]. As a result, 114 GmGRAS genes (except for GmGRAS40, GmGRAS94 and GmGRAS117) were recruited (Additional file 11: Table S10). Moreover, the expression data of 114 GmGRAS genes were $\log _{2}$ normalized to depict a heatmap of GmGRAS genes expression profiles in various tissues (Fig. 9a). According to Fig. 9a, the different GmGRAS gene subfamilies displayed distinct expression patterns. For instance, most GmGRAS genes in the LISCL, SHR and SCL3 subfamilies exhibited low gene expressions in all tissues, however, some genes (GmGRAS67, GmGRAS82 and GmGRAS83 in the LISCL subfamily; GmGRAS97 in the SHR subfamily; GmGRAS15 and GmGRAS65 in the SCL3 subfamily) showed relatively high expression levels in root and nodule. Notably, nine genes (GmGRAS39 and GmGRAS101 in the PAT1 subfamily; GmGRAS11 in the HAM subfamily; GmGRAS50 and GmGRAS64 in the SCL4/7 subfamily; GmGRAS23, GmGRAS32, GmGRAS62 and GmGRAS107 in the DELLA subfamily) presented high expressions in all tissues, which may indicate their crucial functions during soybean plant developments. Interestingly, there were two gene members (GmGRAS20 and GmGRAS104) in the LAS subfamily, and both of them showed high expressions in tissues of leaf, flower, pod, root and early development stages of seed. Besides, most GmGRAS genes exhibited relatively superior gene expressions in the root compared with other tissues. 


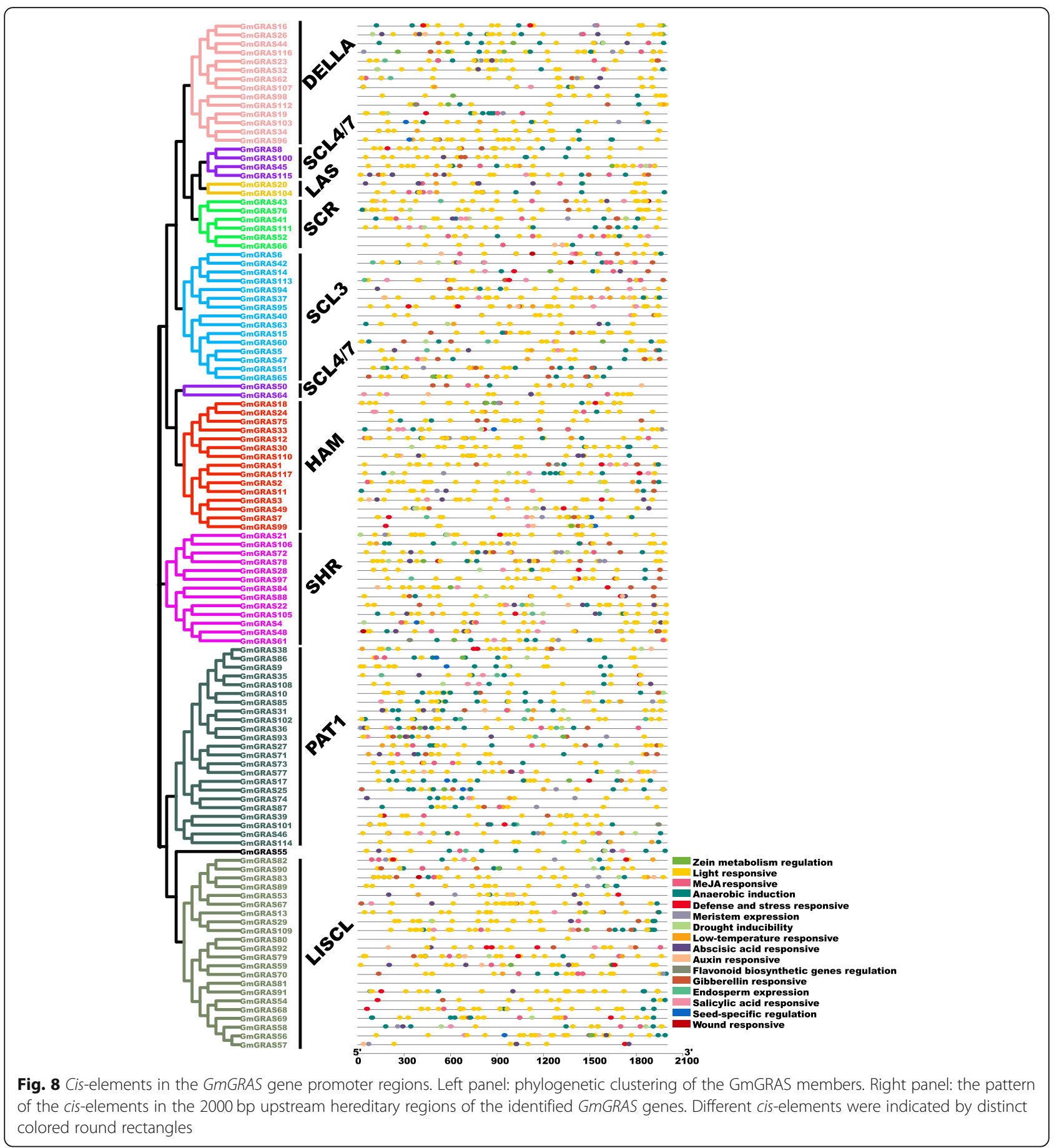

Additionally, we analyzed and calculated the expression correlation coefficients between the 114 GmGRAS genes in Table S11 (Additional file 12). A heatmap was depicted based on the correlation coefficients and the heatmap was further clustered by different GmGRAS subfamilies (Fig. 9b). As is shown in Fig. 9b, the correlation heatmap was divided into diverse blocks with the dotted lines according to the GmGRAS subfamilies. Thus, the correlations among different subfamilies were displayed. To emphasize the inter-correlations of each
GmGRAS subfamily member, we enclosed the subfamilies with the solid boxes and labeled their names in bold. Most GmGRAS genes showed positive correlations with the members of internal or external subfamilies. Whereas, considerable GmGRAS members in the DELLA subfamily tended to exhibit comparatively independent or presented negative correlations with other GmGRAS members. Overall, these results indicated that the functions of GmGRAS genes may be widely correlated and varied in soybean tissues. 


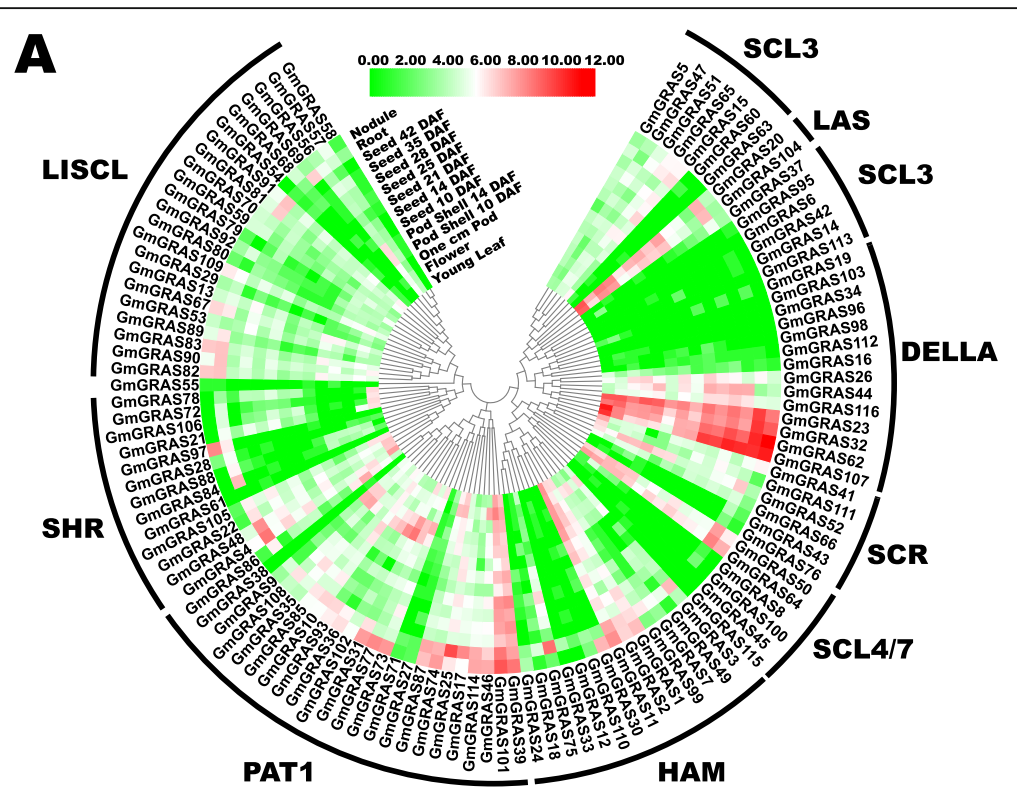

B

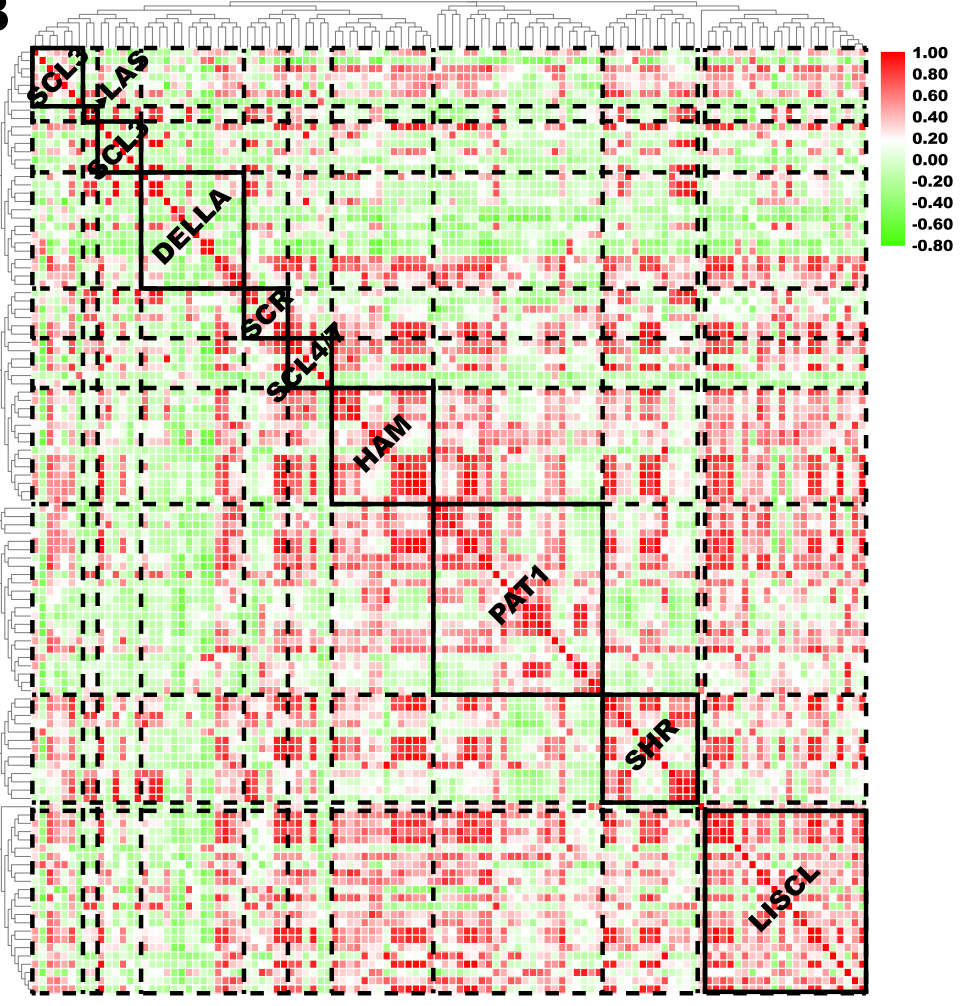

Fig. 9 Expression profiles of the GMGRAS genes in various tissues during soybean plant developments. a Phylogenetically clustered expression profiles of soybean GRAS genes in various tissues during soybean developments based on the public transcriptome data. The RPKM values were displayed for gene expression levels and were $\log _{2}$ normalized to depict the heatmap. DAF: days after flowering. $\mathbf{b}$ Gene expression correlation heatmap of the expressed GmGRAS genes in various tissues during soybean development. Red: positively correlated; green: negatively correlated 
Expression profiles of GmGRAS genes during saline and dehydration stresses and gene expression correlation analyses

Previous studies reported that the GRAS genes were broadly responsive to various abiotic stresses [4, 19, 42]. In the present study, we explored the GmGRAS gene expression profiles in soybean root during saline and dehydration stresses [43]. As a result, 95 members of the identified GmGRAS genes were extracted, and relevant expression information was listed in Table S12 (Additional file 13). Based on the $\log _{2}$ normalized expression data, a heatmap was depicted (Fig. 10a). As is shown in the figure, there were four-time points $(0 \mathrm{~h}, 1 \mathrm{~h}, 6 \mathrm{~h}$ and $12 \mathrm{~h})$ during the abiotic stresses. And the subfamilies both contained the high expression and low expression GmGRAS genes. In particular, most GmGRAS genes in the PAT1 subfamily were at relatively high expression levels. Generally, genes that categorized in the same hereditary clades exhibited similarly high or low transcript levels during saline and dehydration stresses.

Moreover, we carried out the gene expression correlation analyses during saline and dehydration stresses in Table S13 (Additional file 14) and Table S14 (Additional file 15). And two correlation heatmaps were built based on the acquired correlation coefficient matrixes (Fig. 10b and Fig. 10c).

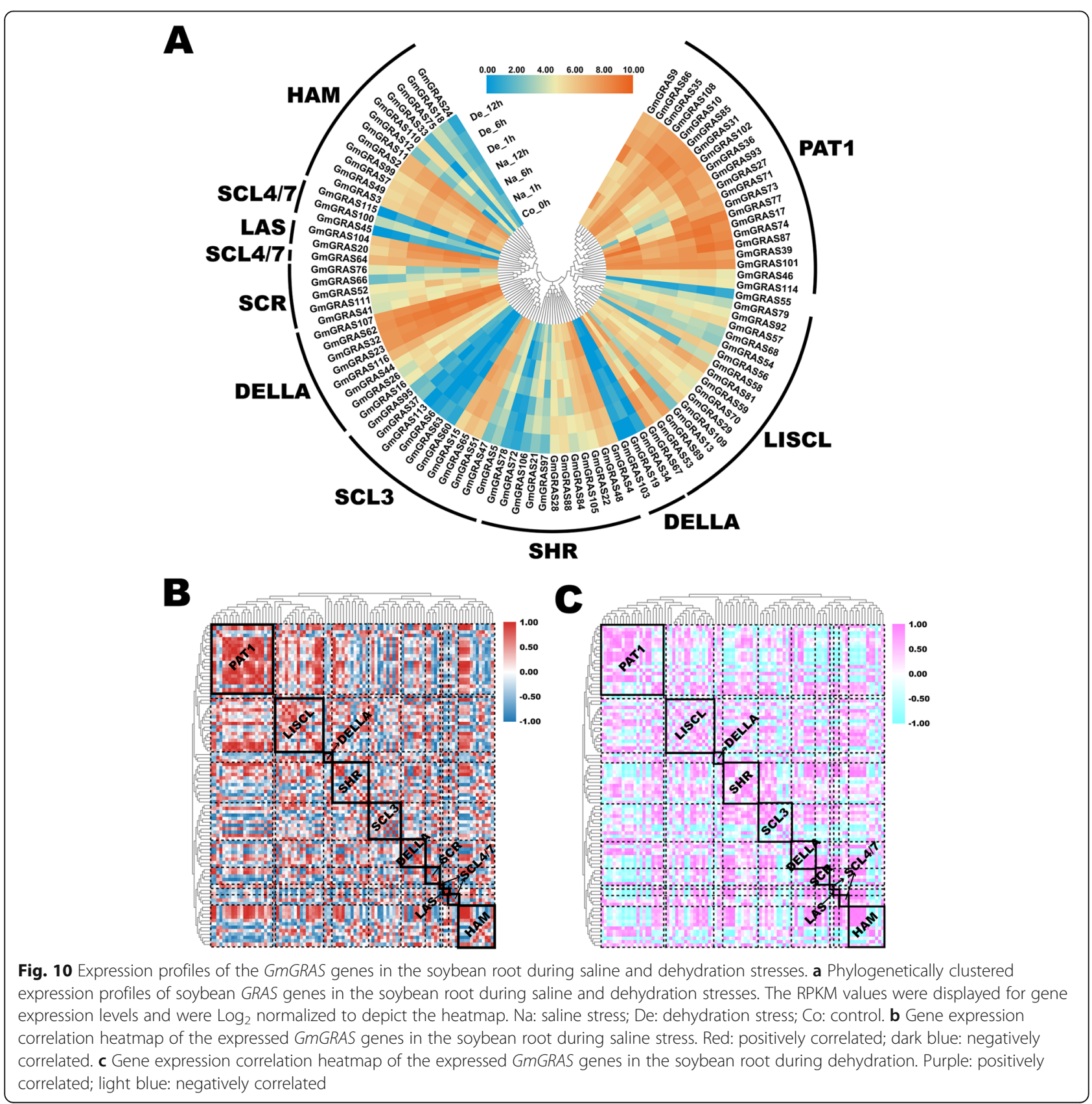


Overall, the positive and negative correlations were universally found and interlaced among the GmGRAS members of internal or external subfamilies both during saline and dehydration stresses. However, the gene expression correlation patterns were distinct during the saline and dehydration stresses. For instance, GmGRAS members in the PAT1 and the SCL3 subfamilies displayed different correlation patterns during saline and dehydration stresses. In all, the different GmGRAS gene members were extensively correlated and diversely expressed during saline and dehydration stresses.

\section{Expression profiles of GmGRAS genes in soybean embryonic axes during seed germination and gene expression correlation analyses}

Seed germination is crucial that influence crop yield and quality. High-vigor soybean embryonic axes were essential to ensure the germination rate [44]. Previous studies paid close attention to the soybean embryonic axis and demonstrated its roles in soybean seed germination from different perspectives $[45,46]$. Besides, the GRAS family genes were also profoundly affected the seed germination [15]. Hence, we analyzed the relevant expression profiles of the GmGRAS genes during soybean germination (Additional file 16: Table S15) [47]. The extracted expression data were $\log _{2}$ normalized to construct a gene expression heatmap (Fig. 11a). As is shown in Fig. 11a, five-time points (dry, 3-HAI (hours after imbibition), 6-HAI, 12-HAI and 24-HAI) were investigated in soybean germination processes. Notably, some soybean GRAS genes had comparatively high gene expressions and displayed time-preference. For instance, GmGRAS73 and GmGRAS77 in the PAT1 subfamily showed high transcriptional levels at the first four-time points and low expressions at 24-HAI. In contrast, the expression of GmGRAS62 in the DELLA subfamily continuously up-regulated and displayed high expression at 24-HAI. Moreover, the extracted expression data were further utilized to construct a heatmap, which was rowscaled with the zero-to-one method to show the expression pattern of each GmGRAS gene during the seed germination (Additional file 17: Fig. S2). In general, different GmGRAS gene members exhibited various expression patterns.

Concomitantly, we conducted the gene expression correlation analyses in Table S16 (Additional file 18) and built the heatmap according to the correlation coefficients of gene expressions (Fig. 11b). As is shown in Fig. 11b, the heatmap was phylogenetically clustered and separated into diverse subunits by red dotted lines to show the correlations among the members of different GmGRAS subfamilies. Moreover, the members of the specific subfamily were highlighted in red solid boxes and annotated with respective subfamily names. On the whole, the GmGRAS genes showed positive or negative correlations both internal and external throughout different GmGRAS subfamilies. In conclusion, the identified GmGRAS genes were broadly correlated and differently expressed during soybean seed germination.

\section{Soybean seed germination assay and quantitative RT-PCR analyses of the representative GmGRAS genes}

For the soybean seed germination assay, five representative soybean embryonic axes from each time point were arrayed by the time axis in Fig. 12a. The sampled soybean embryonic axes were carried out the RNA extraction and the quantitative RT-PCR experiment. To further investigate the potential regulating roles of GmGRAS genes during soybean seed germination, 18 representative GmGRAS genes, whose expression levels were relatively high across different time points (Additional file 16: Table S15), were carefully selected from the identified soybean GRAS genes. The selected genes contained two gene members with diverse expression patterns (Additional file 17: Fig. S2) from each GmGRAS gene subfamily to cover the differently expressed GmGRAS genes as representative as possible. The specific primers of representative GmGRAS genes for the quantitative RT-PCR assay were list in Table S17 (Additional file 19). According to the results of the quantitative RT-PCR experiment, the selected genes in distinct GmGRAS gene subfamilies tended to exhibit different expression patterns (Fig. 12b). Whereas, the selected genes in some subfamilies displayed similar expression patterns. For instance, the expression levels of GmGRAS41 and GmGRAS111 in the SCR subfamily as well as GmGRAS22 and GmGRAS105 in the SHR subfamily were up-regulated from 0-HAI (dry) to 3-HAI and were gradually downregulated from 6-HAI to 24-HAI. Besides, different gene expression patterns also existed in the same subfamily. For example, the expression level of GmGRAS44 in the DELLA subfamily was consecutively down-regulated from 0 -HAI to 24-HAI. In contrast, the expression level of GmGRAS62 was up-regulated from 0-HAI to 6-HAI and was drown-regulated from 12-HAI to 24-HAI. Conclusively, the expression patterns of the representative genes may outline the diverse GmGRAS gene expression tendencies, which highlight the regulations of GmGRAS genes in soybean embryonic axes during seed germination.

\section{Discussion}

The GRAS proteins are plant-specific and play important roles in diverse plant developmental and physiological processes. Recently, the genome-wide identifications of GRAS members in different species have been gradually realized with the rapid development of whole-genome sequencing technologies. Nevertheless, associated studies on the soybean GRAS gene family are still lacking. In the current research, we identified and carried out a comprehensive investigation on the GRAS transcription factors in 


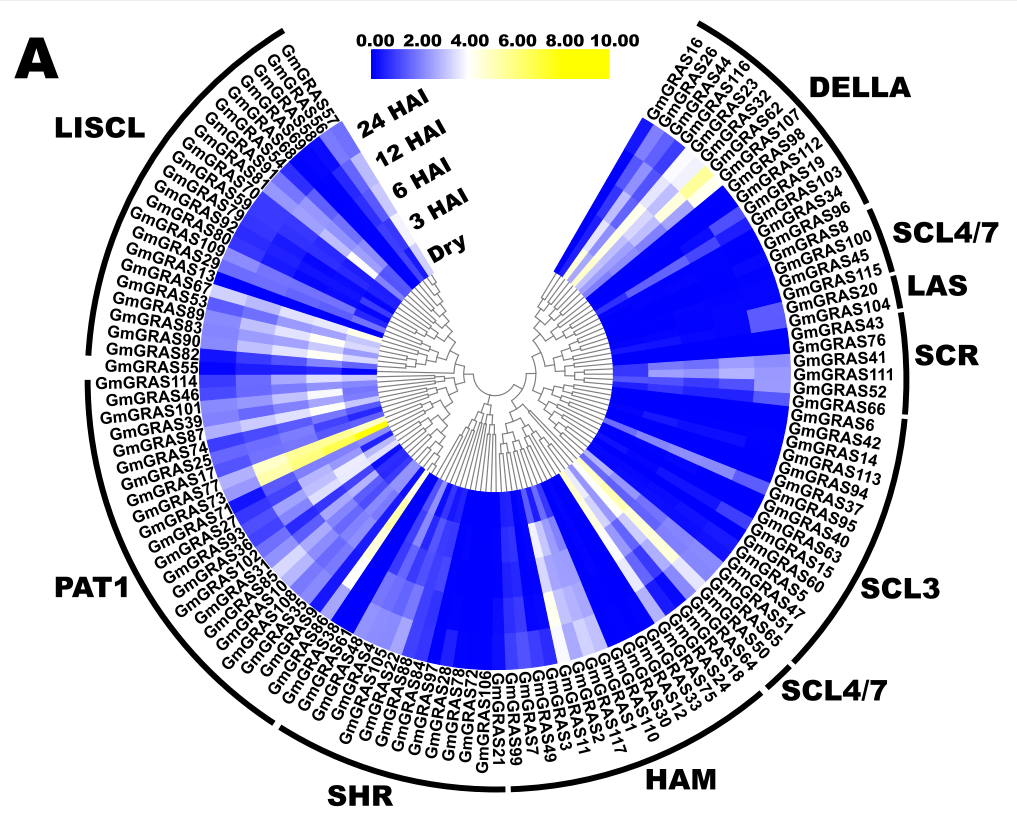

B

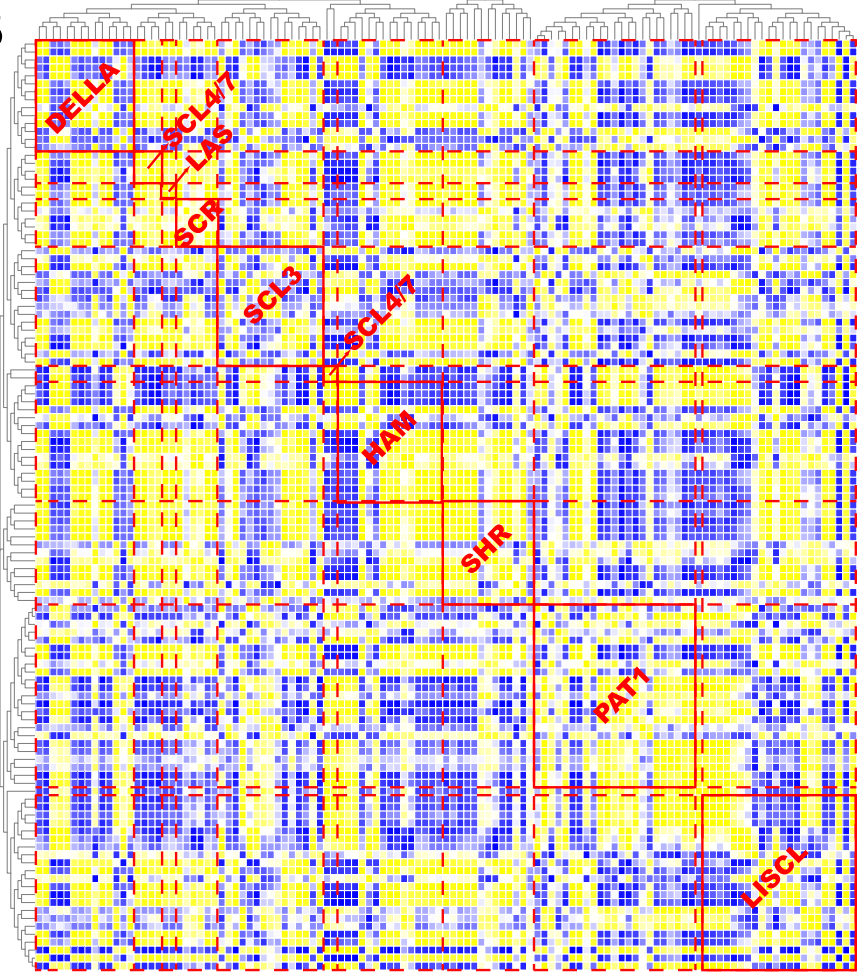

Fig. 11 Expression profiles of the GmGRAS genes in the soybean embryonic axes during seed germination. a Phylogenetically clustered expression profiles of soybean GRAS genes in the soybean embryonic axes during germination based on the reported transcriptome data. The FPKM values were displayed for gene expression levels and were $\log _{2}$ normalized to depict the heatmap. HAl: hours after imbibition. $\mathbf{b}$ Gene expression correlation heatmap of the expressed GmGRAS genes in the soybean embryonic axes during seed germination. Yellow: positively correlated; blue: negatively correlated 


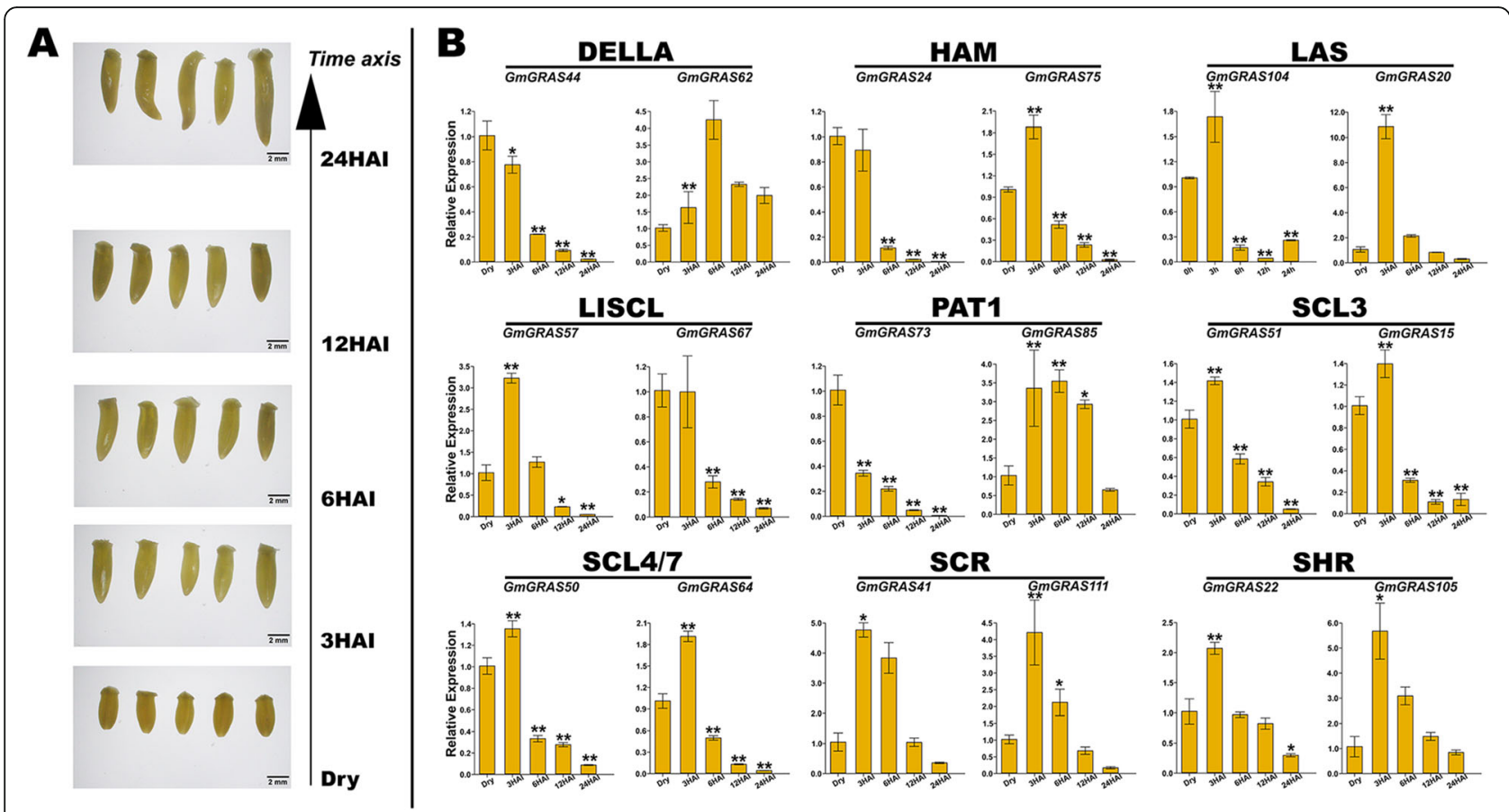

Fig. 12 Soybean seed germination experiment and the quantitative PCR analyses of 18 selected GmGRAS genes in soybean embryonic axes during seed germination. a Photographs of soybean embryonic axes at 0- (Dry), 3-, 6-, 12- and 24-HAl. The size of the soybean embryonic axes can be estimated by using the 2-mm scale at the right bottom. $\mathbf{b}$ Expression patterns of the selected GmGRAS genes in soybean embryonic axes during germination. Data were normalized to the GmActin gene, and vertical bars indicated the standard deviations. The values referred to the mean \pm standard deviation (SD) of three independent biological replicates. Asterisks manifested the corresponding genes significantly up- or down-regulated compared with those in the dry soybean embryonic axes $\left(^{*} P<0.05\right.$, ${ }^{* *} P<0.01$, Student's $t$-test)

soybean, including their phylogenetic relations, gene structures, conserved domains, motif patterns, gene chromosomal distributions, gene duplications, synteny analyses and gene evolutionary analyses. Besides, the expression patterns of the GmGRAS genes in various tissues, under saline and dehydration stresses and during seed germination processes were also explored.

In this investigation, 117 GmGRAS genes were identified. The GRAS members in soybean exceed those in Arabidopsis (32 members) [8], castor beans (48 members) [25], tomato (53 members) [48] and rice (60 members) [8]. This result was also paralleled to the genome size of the species, which may indicate the positive correlations between specific genome size and the number of GRAS members. Phylogenetic analyses divided the GmGRAS proteins into nine subfamilies based on the classifications on AtGRAS (Fig. 1). The GRAS members categorized in the same subfamily or clade may suggest their similar functions in different species.

Previous research demonstrated that the plant GRAS family originated from the prokaryotic genome and horizontally transferred along with gene duplications [49]. Soybean is an ancient polyploid and has a highly duplicated genome, with approximately $75 \%$ of the genes present occurring in multiple copies [50]. According to
Table S6 (Additional file 7), apart from tandem duplications, considerable GmGRAS family members were derived from segmental duplication events. Our results verified that both the segmental and tandem duplications were important contributors to the expansion of the GRAS gene family. Besides, introns were speculated crucial in plant evolutions, which preferred to raise at the earlier stages of gene expansion and gradually lost over time [51, 52]. Nonetheless, there were also exceptions that introns could be larger during evolutions and were considered to be a necessary way to acquire new gene functions $[19,53]$. As is shown in Fig. 2, most detected GmGRAS gene members lacked introns, which parallel to the early reports on the conserved characteristics of GRAS genes $[4,7]$. Notably, most PAT1 subfamily members (16 members) have introns in $5^{\prime}$ UTRs (untranslated regions), which may indicate the PAT1 subfamily is a newly evolved GRAS clade in the soybean genome. The specific domains or motifs of the GRAS proteins ensured the features in protein interaction as well as DNA binding modifications [54]. In the current study, we comprehensively classified the MEME-motifs into the five conserved motifs at the C-terminal of GRAS proteins (Fig. 3b and Fig. 3c). And the MEMEmotifs in the five conserved C-terminal motifs fluctuated 
with diverse subfamilies, which may reflect the distinct biological functions of different GmGRAS subfamily members. In all, the consistencies and divergences of the structures among the GmGRAS members may directly or indirectly illustrate their functional similarities and disparities.

In this investigation, the identified GmGRAS genes unevenly distributed on 20 chromosomes and one scaffold of the soybean genome (Fig. 4). Notably, Chr11 contained the most GmGRAS genes (16 genes), which were mainly originated from the DELLA, HAM, LISCL, SCR, SHR, SCL3 and SCL4/7 subfamilies (Additional file 1: Table S1). In an early study, Sun et al. elaborately summarized the functional polymorphism of plant GRAS domains: the DELLA domain was associated with transcription co-activation, light signalling, gibberellic acid or jasmonic acid signalling, integrator of abiotic stresses, auxin and ethylene signals; the HAM domain was related to nodulation signalling, transcription co-activation in response to auxin, shoot meristem maintenance; the LISCL domain was relevant to transcription activation or co-activation in response to different signals; the SCR domain participated in root or shoot radial patterning; the SHR domain was linked to transcriptional regulation, root or shoot radial patterning and nodulation signalling; the SCL3 domain can act with DELLA and SHR/SCR to mediate cell elongation in root endodermis; the SCL4/7 domain was the transcription regulator in response to environmental stresses [55]. Besides, among the 16 genes, GmGRAS48 from the SHR subfamily, GmGRAS50 from the SCL4/7 subfamily, GmGRAS51 from the SCL3 subfamily, GmGRAS52 from the SCR subfamily, GmGRAS53 and GmGRAS56 from the LISCL subfamily and GmGRAS62 from the DELLA subfamily showed relatively high gene expressions in multiple tissues or organs throughout various developmental stages (Additional file 11: Table S10). Taken together, we speculated that some key GmGRAS genes on Chr11 may take potential roles in regulating soybean plant growth and development.

To explore the evolutionary relationships of GRAS members among different species, we recruited four dicotyledons and two monocotyledons and carried out the synteny analyses. As a result, soybean and Glycine soja exhibited the best orthologous correlations. Comparably, the GRAS members in soybean and Oryza sativa performed the weakest orthologous correlations in the current study. In general, the GRAS members in dicotyledons displayed better synteny with the identified GmGRAS members than those in monocotyledons (Fig. 6). Hence, we speculated that the syntenic correlations among GRAS members may be linked to the evolutionary divergence of the species. Notably, 19 GmGRAS members were found to be syntenic with the GRAS members throughout multiple species (Fig. 7a and Additional file 9: Table S8), which indicated that these orthologous pairs are conserved and may exist before the ancestral divergence [35]. Conclusively, the intersections of the syntenic GRAS members among distinct species may be valuable for conducting relevant explorations on GRAS evolution.

The GRAS members were reported widely participate in regulating plant developments and stress responses $[4,19]$. In the present research, we analyzed the cis-elements in the promoter regions of the detected GmGRAS genes. As is shown in Fig. 8, cis-elements that associate with the plant growth and development broadly existed, such as the light responsive element, auxin responsive element, meristem expression element. We also detected abiotic stress correlated cis-elements, like defense and stress responsive element, drought inducibility element, low temperature responsive element and wound responsive element. Therefore, cis-elements analysis supplied clues for gene function study, especially for relevant genes that respond to different stresses and regulate plant developments.

To seek the expression patterns of GmGRAS genes in various tissues, we extracted and analyzed the transcript levels of the identified GmGRAS genes in different tissues [41]. As a whole, most GmGRAS genes were at relatively low transcription levels, and considerable genes clustered in the same gene subfamilies showed similar expression patterns (Fig. 9a). It is worth noting that some GmGRAS genes, like GmGRAS39 and GmGRAS101 in the PAT1 subfamily displayed high gene expression levels throughout tissues and developmental stages. These GmGRAS genes may be crucial and broadly participate in soybean plant developmental processes. Besides, some GmGRAS genes, like GmGRAS20 and GmGRAS104 in the LAS subfamily exhibited tissues or developmental stages preferences, which highlighted the spatio-temporal regulations of GmGRAS genes during soybean plant growth and developments. Furthermore, according to the gene expression correlation analyses in Fig. 9b, most GmGRAS genes were positively correlated with each other, which demonstrated that the GmGRAS genes widely cooperated in associated regulations. Previously, the DELLA subfamily members in different plants had been extensively studied, which were the key negative regulators of the GA (gibberellic acid) signal transduction pathways and inhibited plant growth and developments $[56,57]$. In this study, most GmGRAS genes in the DELLA subfamily showed negative correlations with other GmGRAS genes or displayed relative independence. Associated with gene expression profiles of the DELLA subfamily genes in Fig. 9a, we speculated that several key genes in the DELLA subfamily may persistently express and regulate soybean growth and developments. Overall, the identified GmGRAS genes exhibited potential functions in regulating soybean plant developments. 
GRAS members are relevant to regulating their biochemical activities in response to abiotic stresses [12]. In this study, the roles of some GmGRAS members during saline and dehydration stresses were investigated [43]. Importantly, considerable GmGRAS genes throughout different GmGRAS gene subfamilies were influenced both by the saline and dehydration stresses. As a whole, the stress responses (high or low gene expression levels) of the identified GmGRAS genes presented similarities during saline and dehydration stresses (Fig. 10a), which may highlight the roles of key GmGRAS genes in response to the abiotic stresses. For instance, most GmGRAS genes in the PAT, LAS and DELLA subfamilies showed high expressions during saline and dehydration stresses (Fig. 10a). Notably, DELLA proteins were reported to play an important role in regulating plant stress tolerance [57], and our investigation further verified the previous findings. Whereas, the gene expression correlation patterns during saline and dehydration stresses were distinctly different (Fig. 10b and Fig. 10c). Hence, we speculated that functional GmGRAS genes may broadly cooperate in response to saline and dehydration stresses under different mechanisms.

Particularly, we investigated the expression profiles of GmGRAS genes in soybean embryonic axes during seed germination [47]. Interestingly, most GmGRAS genes relatively low expressed. However, there were also some high expressed GmGRAS genes in different GmGRAS subfamilies (Fig. 11a). And the GmGRAS genes displayed diverse expression patterns during the seed germination (Additional file 17: Fig. S2). Importantly, as is shown in Fig. 11b, distinct GmGRAS genes were universally correlated, which indicated the functional GmGRAS genes may broadly interact and concertedly affect soybean seed germination. Furthermore, we selected 18 representative GmGRAS genes and carried out the quantitative RTPCR analyses. Compared to the reported expression patterns of GmGRAS genes in Fig. S2 (Additional file 17), GmGRAS24, GmGRAS41, GmGRAS44, GmGRAS50, GmGRAS51, GmGRAS57, GmGRAS64, GmGRAS67, GmGRAS73 and GmGRAS75 that validated by the quantitative RT-PCR assay showed fundamental uniformity (Fig. 12b). Whereas, GmGRAS15, GmGRAS20, GmGRAS22, GmGRAS62, GmGRAS85, GmGRAS104, GmGRAS105 and GmGRAS104 displayed different expression patterns from those of the reported transcriptome data [47]. For instance, in the transcriptome data, the transcript levels of GmGRAS62 was gradually up-regulated from 0-HAI to 24-HAI (Additional file 17: Fig. S2). However, in the quantitative RT-PCR assay result, the expression levels GmGRAS62 was first up-regulated from 0-HAI to 6-HAI then was down-regulated from 6-HAI to 24-HAI (Fig. 12b). For the soybean seed gemination assay in the current research, we added the seed presoaking process compared to the early study [47], which accelerated soybean seed imbibition. And this may cause the preceding expression of relevant GmGRAS genes. Another possible cause may due to the differences between testing soybean varieties in the present study (cv. 'Williams 82') and the previous research (cv. 'BRS 284') [47]. In summary, GmGRAS genes have potential regulatory roles during soybean seed germination.

This study provided a systematic investigation of GRAS genes in soybean, which may be beneficial to gain insights into their biological functions. However, the current study only provided a preliminary characterization of GmGRAS genes and further functional validation should be carried out to understanding the different roles of GmGRAS genes in various biological processes.

\section{Conclusions}

In this study, we identified 117 GmGRAS genes in soybean. These GmGRAS genes unevenly located on 20 chromosomes and one scaffold in the soybean genome. The identified GmGRAS members were further classified into nine GmGRAS subfamilies. Gene structure analyses turned out that most GmGRAS genes lack introns, suggesting that the structures of GmGRAS genes were highly conserved. Conserved domain and motif pattern analyses showed that the GRAS members in the same subfamily or clade displayed broadly similarities, which may indicate their parallel gene functions. Moreover, the emergence of new GmGRAS genes mainly drove by gene duplications, and segmental duplication events took the lead in GmGRAS gene family expansion. Besides, ciselement and gene expression analyses revealed the potential regulations of the identified GmGRAS genes in various tissues, during saline and dehydration stresses and during soybean seed germination processes. In all, we comprehensively investigated the characteristics of GRAS genes in soybean, and the results provided valuable clues for understanding the gene biological functions and future studies on GmGRAS genes.

\section{Methods \\ Mining of GRAS family members in soybean}

For the identification of GRAS genes in soybean (soybean Wm82.a2.v1 genome version), we obtained all the AtGRAS protein sequences as the query sequences from the TAIR database (https://www.arabidopsis.org/) [4, 58]. The soybean genome and genome annotation files were downloaded from Phytozome v12. 1.6 database (https://phytozome.jgi.doe.gov/ pz/portal.html). Based on these data, we extracted the most representative GmGRAS member sequences by using TBtools software [59], and GmGRAS protein sequences were further queried and verified in the NCBI protein database by BLASTp (https://blast.ncbi.nlm.nih.gov/Blast.cgi? PROGRAM = blastp\&PAGE_TYPE $=$ BlastSearch\&LINK_

LOC $=$ blasthome). For the domain composition analyses, we used the NCBI-Conserved Domain database (https://www. ncbi.nlm.nih.gov/Structure/cdd/wrpsb.cgi). Proteins that lack 
GRAS associated domains were manually deleted. Moreover, protein sequences with obvious errors in their gene length or less than 100 aa (amino acid) were also removed. The ExPASy website (http://expasy.org/tools/) was employed for evaluations of molecular weight (MW), isoelectric point (pI) and amino acid numbers of the identified GmGRAS proteins. For GmGRAS gene subcellular localization predictions, we used the CELLO (http://cello.life.nctu.edu.tw/) online tools.

\section{Phylogenetic analyses and classifications of the GmGRAS proteins}

The GRAS protein sequences of Arabidopsis and soybean were together aligned by using the muscle method of MEGA 7.0 (https://www.megasoftware.net/) with the default parameters. The aligned sequences were followed by the neighbor-joining $(\mathrm{NJ})$ method to build the phylogenetic tree, with the following parameters: Poisson model, pairwise deletion, and 1000 bootstrap replications [35]. The identified GmGRAS proteins were further categorized into different subfamilies based on the records of AtGRAS subfamily members in the TAIR database (https://www.arabidopsis.org/). The modified phylogenetic tree was depicted by FigTree v1.4.3 (http://tree.bio.ed.ac.uk/software/figtree/) and Adobe Illustrator CC 2019 (https:/www.adobe.com/ products/illustrator/free-trial-download.html).

\section{Gene structures and conserved motif analyses}

The gene structures were depicted by TBtools [59] with the GFF3 file of the soybean genome. The conserved motifs scanning of GmGRAS proteins were conducted by MEME v5.1.1 (http://meme-suite.org/tools/meme) with 20 MEME-motifs shown in the result. The visualization and the Seq Logos of the MEME-motifs were created by TBtools. Referring to the classifications of Quan et al. on the GRAS C-terminal conserved motifs, the identified MEME-motifs were further categorized into the specific LHR I, VHIID, LHR II, PFYRE and SAW motifs [34]. The output graphs were modified by Adobe Illustrator CC 2019.

\section{GmGRAS gene chromosomal locations, duplications and synteny analyses}

The chromosomal locations and duplications of GmGRAS genes were mapped according to the available soybean genome information on Phytozome and displayed by the TBtools software [59]. The gene density information of each chromosome or scaffold was calculated by TBtools. To explore the synteny relationships of the orthologous GRAS genes among soybean and other species, we additionally downloaded the genome data and the gene annotation files of Arabidopsis thaliana (TAIR annotation release 10), Glycine soja (V1.1), Vigna unguiculata (V1.1), Solanum lycopersicum (ITAG3.2), Oryza sativa (MSU annotation release 7.0) and Sorghum bicolor) (V3.2) from Phytozome. The syntenic analyzing graphs were constructed by using the Dual Synteny Plotter function in TBtools. The Venn diagram of the syntenic GRAS genes throughout diverse species was depicted by TBtools. Nonsynonymous substitution (Ka) and synonymous substitution (Ks) of the duplicated GRAS genes were calculated by TBtools. The output graphs were modified by Adobe Illustrator CC 2019.

\section{Cis-element analyses of GmGRAS genes}

The upstream $2000 \mathrm{bp}$ sequences of the identified GmGRAS genes were extracted by TBtools [59]. Then the extracted sequences were submitted to PlantCARE website (http://bioinformatics.psb.ugent.be/webtools/ plantcare/html/) to predict the cis-elements in promoter regions. The diagram of cis-elements of GmGRAS genes was displayed by TBtools and was modified by Adobe Illustrator CC 2019.

\section{Expression profile analyses of GmGRAS genes}

The transcriptional levels of the identified GmGRAS genes in different tissues were retrieved from the transcriptome data on SoyBase (https://soybase.org/soyseq/) [41], which were assessed by the RPKM (reads per kilobase million) values. The expression profiles of GmGRAS genes during saline and dehydration stresses were obtained by the RPKM values from the published research data [43]. The transcriptional levels of the GmGRAS genes in soybean embryonic axes during germination were extracted from an early study of Bellieny-Rabelo et al., which were scored by the FPKM (fragments per kilobase million) values [47]. Heatmaps of the GmGRAS gene expressions were illustrated based on the $\log _{2}$ normalized RPKM or FPKM values by using TBtools [59]. To explore the GmGRAS gene expression correlations, both the extracted RPKM and FPKM values were submitted and calculated on Omicshare online tools (https://www.omicshare.com/tools/Home/Soft/getsoft).

Besides, the correlation heatmaps of GmGRAS gene expressions were phylogenetically clustered and depicted by TBtools. The output graphs were modified by Adobe Illustrator CC 2019.

\section{Plant material and soybean seed germination assay}

Soybean cultivar Williams 82, a well-known soybean genome referencing variety, was used in this study. Three independent soybean plants were randomly selected and harvested the seeds for three independent biological replicates in this study. All the testing soybean seeds were harvested in the autumn of 2019 at the Dangtu Experimental Station, National Center for Soybean Improvement, Nanjing Agricultural University, Dangtu, Anhui, China. Referring to the previous study, 
we focused five-time points during soybean seed germination: dry, 3-HAI (hours after imbibition), 6-HAI, 12HAI and 24-HAI [47]. Importantly, we adopted the germination pouches (medium size, $18 \mathrm{~cm}$ height and $12.5 \mathrm{~cm}$ width, PhenoTrait Technology Co., Ltd.) to conduct the soybean seed germination experiment. For each time point, 40 healthy seeds were selected from the independent soybean plant harvesting seeds. And the seeds were placed in a $40^{\circ} \mathrm{C}$ drying oven for 3 days to ensure the uniformity of seed moisture. The selected seeds were then disinfected by $0.05 \%$ potassium permanganate solution for $5 \mathrm{~min}$ and washed with deionized water. According to the instruction book of the germination pouch, the disinfected soybean seeds were soaked in deionized water for 2 hours to accelerate seed imbibition and ensure seed germination. Next, we placed eight seeds in each germination pouch and added $15 \mathrm{~mL}$ deionized water. Then germination pouches were put on the germination pouch shelf and placed in the dark in a temperature-controlled incubator at $25^{\circ} \mathrm{C}$. Embryonic axes were carefully separated from cotyledons for RNA extraction at each time point. Five representative seed embryonic axes at each time point were photographed by using a SPOT-RT digital camera (Diagnostic Instruments, Sterling Heights, MI) and an OLYMPUS SZ61 stereomicroscope (Olympus, Melville, NY, USA).

\section{RNA isolations and quantitative RT-PCR analyses}

To validate the expression patterns in embryonic axes during soybean seed germination, we selected 18 representative GmGRAS genes for the quantitative RT-PCR analyses. Three independent biological replicates contained three independent plants were used for quantitative RT-PCR. The specific quantitative RT-PCR primers of the selected GmGRAS genes were designed by Primer Premier 5 and listed in Table S17 (Additional file 19). Referring to the former research of Bellieny-Rabelo et al., 20 healthy seed embryonic axes were randomly selected from the 40 embryonic axes of each independent plant each time point for total RNA isolations [47]. The total RNA was extracted by using the RNAprep pure plant kit (TIANGEN, Beijing, China) from the frozen embryonic axes. All RNA was analyzed by electrophoresis and then quantified with a Nanodrop ND-1000 spectrophotometer (Nanodrop, Wilmington, DE, USA). The HiScript II 1st Strand cDNA Synthesis Kit (Vazyme Biotech, Nanjing, China) was adopted to remove the genomic DNA and to convert the total RNA to cDNA [60]. The SYBR qPCR Master Mix (Vazyme Biotech, Nanjing, China) was adopted to conduct the quantitative RT-PCR assay on a BioRad CFX96 real-time system [60]. The housekeeping GmActin gene was determined as an internal control. Triplicate quantitative assays were performed on each cDNA sample and analyzed by a $2^{-\triangle \Delta C T}$ method [61].

\section{Statistical analyses}

Student's $t$-test was performed by using by Graphpad Prism 8 (https://www.graphpad.com/scientific-software/ prism/). Test differences were determined to be significant with a $P$-value cut-off of 0.05 . All the error bars were standard deviation (SD) from the independent biological replicates.

\section{Supplementary information}

Supplementary information accompanies this paper at https://doi.org/10. 1186/s12870-020-02636-5.

Additional file 1: Table S1. The 117 identified GmGRAS genes in this study.

Additional file 2: Table S2. Coding sequences and protein sequences of the identified soybean GRAS gene members.

Additional file 3: Table S3. Corresponding names of 32 AtGRAS genes in Arabidopsis.

Additional file 4: Table S4. Analyses the motifs in soybean GRAS proteins from the MEME website.

Additional file 5: Figure S1. Seq Logos of 20 MEME-motifs for the identified GmGRAS proteins.

Additional file 6: Table S5. Gene density of each chromosome or scaffold of the soybean genome.

Additional file 7: Table S6. Tandemly and segmentally duplicated GmGRAS gene pairs.

Additional file 8: Table S7. One-to-one orthologous relationships between the GRAS gene members in soybean and the other six species.

Additional file 9: Table S8. Non-redundant GmGRAS gene IDs associated with the syntenic relationships between soybean and the other six species.

Additional file 10: Table S9. Cis-element analyses of the GmGRAS gene promoter regions.

Additional file 11: Table S10. Expression profiles of GMGRAS genes in multiple tissues throughout various developmental stages.

Additional file 12: Table S11. Pairwise correlation coefficients between different expressed GmGRAS genes in various tissues.

Additional file 13: Table S12. Expression profiles of GmGRAS genes in soybean root during dehydration and salt stresses.

Additional file 14: Table S13. Pairwise correlation coefficients between different expressed GmGRAS genes during saline stress.

Additional file 15: Table S14. Pairwise correlation coefficients between different expressed GMGRAS genes during dehydration stress.

Additional file 16: Table S15. Expression profiles of GmGRAS genes in soybean embryonic axes during seed germination.

Additional file 17: Figure S2. Phylogenetically clustered expression patterns of the expressed GmGRAS genes in soybean embryonic axes during germination based on the reported transcriptome data. The FPKM value was row-scaled with the zero-to-one method to show the expression pattern of each GMGRAS gene during the seed germination.

Additional file 18: Table S16. Pairwise correlation coefficients between different expressed GmGRAS genes in soybean embryonic axes during seed germination.

Additional file 19: Table S17. Sequences of the primers used in this study.

\section{Abbreviations}

Gm: Glycine max; TFs: Transcription factors; GAl: Gibberellic acid insensitive; RGA: Repressor of GA1-3 mutant; SCR: Scarecrow; LHRI: Leucine-rich region I; LHRII: Leucine-rich region II; Va: Vitis amurensis; At: Arabidopsis thaliana; phyA: Phytochrome A; Mt: Medicago truncatula; aa: Amino acid; 
MW: Molecular weight; pl: Isoelectric point; NJ: Neighbor-joining; Ka: Nonsynonymous substitution; Ks: Synonymous substitution; RPKM: Reads per kilobase million; FPKM: Fragments per kilobase million; HAl: Hours after imbibition; SD: Standard deviation; ORF: Open reading frame; CDS: Coding sequence; Chr: Chromosome; kb: Kilobase; Gs: Glycine soja; Vu: Vigna unguiculate; SI: Solanum lycopersicum; Sb: Sorghum bicolor; Os: Oryza sativa; cm: Centimeter; DAF: Days after flowering; UTRs: Untranslated regions

\section{Acknowledgments}

Not applicable.

\section{Authors' contributions}

SPY and LW conceived and designed the research. LW conducted the data analysis, experiments, and finished the original manuscript. LW, XLD and YQG carried out the quantitative RT-PCR analyses. LW and SPY wrote and revised the manuscript. All the authors read and approved the final manuscript.

\section{Funding}

This study was supported by grants from the National Key R\&D Program of China (2016YFD0101500, 2016YFD0101504), the Fundamental Research Funds for the Central Universities (KYT201801), and the Program for Changjiang Scholars and Innovative Research Team in University (PCSI RT_17R55)

\section{Availability of data and materials}

All data generated or analyzed during this study are included in this published article and its Additional files.

\section{Ethics approval and consent to participate}

Not applicable.

\section{Consent for publication}

Not applicable.

\section{Competing interests}

The authors declare that they have no competing interests.

Received: 24 June 2020 Accepted: 30 August 2020

Published online: 05 September 2020

\section{References}

1. Wang YX, Liu ZW, Wu ZJ, Li H, Wang WL, Cui X, Zhuang J. Genome-wide identification and expression analysis of GRAS family transcription factors in tea plant (Camellia sinensis). Sci Rep. 2018;8(1):3949.

2. Ho-Plágaro T, Molinero-Rosales N, Fariña Flores D, Villena Díaz M, GarcíaGarrido JM. Identification and expression analysis of GRAS transcription factor genes involved in the control of arbuscular mycorrhizal development in tomato. Front Plant Sci. 2019;10:268.

3. Fan S, Zhang D, Gao C, Zhao M, Wu HQ, Li YM, Shen YW, Han MY. Identification, classification, and expression analysis of GRAS gene family in Malus domestica. Front Physiol. 2017:8:253.

4. Liu MY, Huang L, Ma ZT, Sun WJ, Wu Q, Tang ZZ, Bu TL, Li CL, Chen H. Genome-wide identification, expression analysis and functional study of the GRAS gene family in Tartary buckwheat (Fagopyrum tataricum). BMC Plant Biol. 2019;19(1):342.

5. Pysh LD, Wysocka-Diller JW, Camilleri C, Bouchez D, Benfey PN. The GRAS gene family in Arabidopsis: sequence characterization and basic expression analysis of the SCARECROW-LIKE genes. Plant J. 1999;18(1):111-9.

6. Bolle C. The role of GRAS proteins in plant signal transduction and development. Planta. 2004;218(5):683-92.

7. Chen Y, Zhu PP, Wu SY, Lu Y, Sun J, Cao QH, Li ZY, Xu T. Identification and expression analysis of GRAS transcription factors in the wild relative of sweet potato Ipomoea trifida. BMC Genomics. 2019;20(1):911.

8. Tian CG, Wan P, Sun SH, Li JY, Chen MS. Genome-wide analysis of the GRAS gene family in rice and Arabidopsis. Plant Mol Biol. 2004:54(4):519-32.

9. Lee MH, Kim B, Song SK, Heo JO, Yu NL, Lee SA, Kim M, Kim DG, Sohn SO, Lim CE, et al. Large-scale analysis of the GRAS gene family in Arabidopsis thaliana. Plant Mol Biol. 2008;67(6):659-70.
10. Bolle C, Koncz C, Chua NH. PAT1, a new member of the GRAS family, is involved in phytochrome a signal transduction. Genes Dev. 2000;14(10): 1269-78.

11. Greb T, Clarenz O, Schafer E, Muller D, Herrero R, Schmitz G, Theres K. Molecular analysis of the LATERAL SUPPRESSOR gene in Arabidopsis reveals a conserved control mechanism for axillary meristem formation. Genes Dev. 2003;17(9):1175-87.

12. Niu YL, Zhao TT, Xu XY, Li JF. Genome-wide identification and characterization of GRAS transcription factors in tomato (Solanum lycopersicum). PeerJ. 2017;5:e3955.

13. Li P, Zhang B, Su TB, Li PR, Xin XY, Wang WH, Zhao XY, Yu YJ, Zhang DS, Yu SC, et al. BrLAS, a GRAS transcription factor from Brassica rapa, is involved in drought stress tolerance in transgenic Arabidopsis. Front Plant Sci. 2018;9:1792.

14. Hirsch S, Oldroyd GED. GRAS-domain transcription factors that regulate plant development. Plant Signal Behav. 2009:4(8):698-700.

15. Tyler L, Thomas SG, Hu JH, Dill A, Alonso JM, Ecker JR, Sun TP. DELLA proteins and gibberellin-regulated seed germination and floral development in Arabidopsis. Plant Physiol. 2004;135(2):1008-19.

16. Heo JO, Chang KS, Kim IA, Lee MH, Lee SA, Song SK, Lee MM, Lim J. Funneling of gibberellin signaling by the GRAS transcription regulator SCAR ECROW-LIKE 3 in the Arabidopsis root. P Natl Acad Sci USA. 2011;108(5): 2166-71.

17. Yoshida H, Ueguchi-Tanaka M. DELLA and SCL3 balance gibberellin feedback regulation by utilizing INDETERMINATE DOMAIN proteins as transcriptional scaffolds. Plant Signal Behav. 2014;9(9):e29726.

18. Cui HC, Levesque MP, Vernoux T, Jung JW, Paquette AJ, Gallagher KL, Wang JY, Blilou I, Scheres B, Benfey PN. An evolutionarily conserved mechanism delimiting SHR movement defines a single layer of endodermis in plants. Science. 2007;316(5823):421-5.

19. Zhang B, Liu J, Yang ZE, Chen EY, Zhang CJ, Zhang XY, Li FG. Genome-wide analysis of GRAS transcription factor gene family in Gossypium hirsutum L. BMC Genomics. 2018;19(1):348.

20. Yuan YY, Fang LC, Karungo SK, Zhang LL, Gao YY, Li SH, Xin HP. Overexpression of VaPAT1, a GRAS transcription factor from Vitis amurensis, confers abiotic stress tolerance in Arabidopsis. Plant Cell Rep. 2016;35(3):655-66.

21. Torres-Galea $P$, Huang LF, Chua NH, Bolle C. The GRAS protein SCL13 is a positive regulator of phytochrome-dependent red light signaling, but can also modulate phytochrome a responses. Mol Gen Genomics. 2006;276(1):13-30.

22. Hirsch S, Kim J, Muñoz A, Heckmann AB, Downie JA, Oldroyd GED. GRAS proteins form a DNA binding complex to induce gene expression during nodulation signaling in Medicago truncatula. Plant Cell. 2009;21(2):545-57.

23. Stuurman J, Jäggi $F$, Kuhlemeier C. Shoot meristem maintenance is controlled by a GRAS-gene mediated signal from differentiating cells. Genes Dev. 2002;16(17):2213-8.

24. Liu XY, Widmer A. Genome-wide comparative analysis of the GRAS gene family in Populus, Arabidopsis and rice. Plant Mol Biol Report. 2014;32(6): 1129-45.

25. Xu W, Chen ZX, Ahmed N, Han B, Cui QH, Liu AZ. Genome-wide identification, evolutionary analysis, and stress responses of the GRAS gene family in castor beans. Int J Mol Sci. 2016;17(7):1004.

26. Guo YY, Wu HY, Li X, Li Q, Zhao XY, Duan XQ, An YR, Lv W, An HL. Identification and expression of GRAS family genes in maize (Zea mays L.) PLoS One. 2017;12(9):e0185418.

27. Wang L, Cheng YB, Ma QB, Mu YH, Huang ZF, Xia QJ, Zhang GY, Nian H. QTL fine-mapping of soybean (Glycine max L.) leaf type associated traits in two RILs populations. BMC Genomics. 2019;20(1):260.

28. Bencke-Malato M, Cabreira C, Wiebke-Strohm B, Bücker-Neto L, Mancini E, Osorio MB, Homrich MS, Turchetto-Zolet AC, De Carvalho MCCG, Stolf R, et al. Genome-wide annotation of the soybean WRKY family and functional characterization of genes involved in response to Phakopsora pachyrhizi infection. BMC Plant Biol. 2014;14(1):236.

29. Du H, Yang SS, Liang Z, Feng BR, Liu L, Huang YB, Tang YX. Genome-wide analysis of the MYB transcription factor superfamily in soybean. BMC Plant Biol. 2012;12:106.

30. Le DT, Nishiyama R, Watanabe Y, Mochida K, Yamaguchi-Shinozaki K, Shinozaki K, Tran LSP. Genome-wide survey and expression analysis of the plant-specific NAC transcription factor family in soybean during development and dehydration stress. DNA Res. 2011;18(4):263-76.

31. Chen X, Chen Z, Zhao HL, Zhao Y, Cheng BJ, Xiang Y. Genome-wide analysis of soybean HD-zip gene family and expression profiling under salinity and drought treatments. PLoS One. 2014;9(2):e8715. 
32. Ha CV, Le DT, Nishiyama R, Watanabe Y, Sulieman S, Tran UT, Mochida K Dong NV, Yamaguchi-Shinozaki K, Shinozaki K, et al. The auxin response factor transcription factor family in soybean: genome-wide identification and expression analyses during development and water stress. DNA Res. 2013;20(5):511-24.

33. Fan CM, Wang X, Wang YW, Hu RB, Zhang XM, Chen JX, Fu YF. Genomewide expression analysis of soybean MADS genes showing potential function in the seed development. PLoS One. 2013;8(4):e62288.

34. Quan SW, Niu JX, Zhou L, Xu H, Ma L, Qin Y. Genome-wide identification, classification, expression and duplication analysis of GRAS family genes in Juglans regia L. Sci Rep. 2019;9(1):11643.

35. Xie T, Chen CJ, Li CH, Liu JR, Liu CY, He YH. Genome-wide investigation of WRKY gene family in pineapple: evolution and expression profiles during development and stress. BMC Genomics. 2018;19(1):490.

36. Holub EB. The arms race is ancient history in Arabidopsis, the wildflower. Nat Rev Genet. 2001;2(7):516-27.

37. Cannon SB, Mitra A, Baumgarten A, Young ND, May G. The roles of segmental and tandem gene duplication in the evolution of large gene families in Arabidopsis thaliana. BMC Plant Biol. 2004;4(1):10

38. Zhu Y, Wu NN, Song WL, Yin GJ, Qin YJ, Yan YM, Hu YK. Soybean (Glycine max) expansin gene superfamily origins: segmental and tandem duplication events followed by divergent selection among subfamilies. BMC Plant Biol. 2014;14(1):93.

39. Yu J, Wang J, Lin W, Li SG, Li H, Zhou J, Ni PX, Dong W, Hu SN, Zeng CQ, et al. The genomes of Oryza sativa: a history of duplications. PLoS Biol. 2005;3(2):e38.

40. Wang W, Jiang W, Liu JG, Li Y, Gai JY, Li Y. Genome-wide characterization of the aldehyde dehydrogenase gene superfamily in soybean and its potential role in drought stress response. BMC Genomics. 2017;18(1):518.

41. Severin AJ, Woody JL, Bolon YT, Joseph B, Diers BW, Farmer AD, Muehlbauer GJ, Nelson RT, Grant D, Specht JE, et al. RNA-Seq atlas of Glycine max: a guide to the soybean transcriptome. BMC Plant Biol. 2010;10:160.

42. Shan ZY, Luo XL, Wu MY, Wei LM, Fan ZP, Zhu YM. Genome-wide identification and expression of GRAS gene family members in cassava. BMC Plant Biol. 2020;20(1):46.

43. Belamkar V, Weeks NT, Bharti AK, Farmer AD, Graham MA, Cannon SB. Comprehensive characterization and RNA-Seq profiling of the HD-zip transcription factor family in soybean (Glycine max) during dehydration and salt stress. BMC Genomics. 2014;15(1):950.

44. Simontacchi M, Caro A, Fraga CG, Puntarulo S. Oxidative stress affects [alpha]- tocopherol content in soybean embryonic axes upon imbibition and following germination. Plant Physiol. 1993;103(3):949-53.

45. Gazara RK, de Oliveira EAG, Rodrigues BC, Nunes da Fonseca R, AEA O, Venancio TM. Transcriptional landscape of soybean (Glycine max) embryonic axes during germination in the presence of paclobutrazol, a gibberellin biosynthesis inhibitor. Sci Rep. 2019;9(1):9601.

46. Sangi S, Santos MLC, Alexandrino CR, Da Cunha M, Coelho FS, Ribeiro GP, Lenz D, Ballesteros H, Hemerly AS, Venâncio TM, et al. Cell wall dynamics and gene expression on soybean embryonic axes during germination. Planta. 2019;250(4):1325-37.

47. Bellieny-Rabelo D, De Oliveira EAG, Ribeiro ES, Costa EP, Oliveira AEA, Venancio TM. Transcriptome analysis uncovers key regulatory and metabolic aspects of soybean embryonic axes during germination. Sci Rep. 2016;6: 36009.

48. Huang W, Xian ZQ, Kang X, Tang N, Li ZG. Genome-wide identification, phylogeny and expression analysis of GRAS gene family in tomato. BMC Plant Biol. 2015;15:209.

49. Zhang DP, lyer LM, Aravind L. Bacterial GRAS domain proteins throw new light on gibberellic acid response mechanisms. Bioinformatics. 2012;28(19): 2407-11.

50. Schmutz J, Cannon SB, Schlueter J, Ma JX, Mitros T, Nelson W, Hyten DL, Song QJ, Thelen JJ, Cheng JL, et al. Genome sequence of the palaeopolyploid soybean. Nature. 2010;463(7278):178-83.

51. William Roy S, Gilbert W. The evolution of spliceosomal introns: patterns, puzzles and progress. Nat Rev Genet. 2006;7(3):211-21.

52. Roy SW, Penny D. A very high fraction of unique intron positions in the intron-rich diatom Thalassiosira pseudonana indicates widespread intron gain. Mol Biol Evol. 2007;24(7):1447-57.

53. Iwamoto M, Maekawa M, Saito A, Higo H, Higo K. Evolutionary relationship of plant catalase genes inferred from exon-intron structures: isozyme divergence after the separation of monocots and dicots. Theor Appl Genet. 1998;97(1):9-19.
54. Liu LS, White MJ, MacRae TH. Transcription factors and their genes in higher plants. Eur J Biochem. 1999;262(2):247-57.

55. Sun $X L$, Xue B, Jones WT, Rikkerink E, Dunker AK, Uversky VN. A functionally required unfoldome from the plant kingdom: intrinsically disordered $\mathrm{N}$ terminal domains of GRAS proteins a re involved in molecular recognition during plant development. Plant Mol Biol. 2011;77(3):205-23.

56. Wang YJ, Deng DX. Molecular basis and evolutionary pattern of GA-GID ${ }_{1}-$ DELLA regulatory module. Mol Gen Genomics. 2014;289(1):1-9.

57. Achard P, Genschik P. Releasing the brakes of plant growth: how GAs shutdown DELLA proteins. J Exp Bot. 2009;60(4):1085-92.

58. Khan N, Fatima F, Haider MS, Shazadee H, Liu ZJ, Zheng T, Fang JG. Genome-wide identification and expression profiling of the polygalacturonase (PG) and pectin methylesterase (PME) genes in grapevine (Vitis vinifera L.). Int J Mol Sci. 2019;20(13):3180.

59. Chen CJ, Chen H, Zhang Y, Thomas HR, Frank MH, He YH, Xia R. TBtools - an integrative toolkit developed for interactive analyses of big biological data. Mol Plant. 2020;13(8):1194-202.

60. Li C, Liu XY, Ruan H, Zhang JY, Xie FB, Gai JY, Yang SP. GmWRKY45 enhances tolerance to phosphate starvation and salt stress, and changes fertility in transgenic Arabidopsis. Front Plant Sci. 2020;10:1714.

61. Livak KJ, Schmittgen TD. Analysis of relative gene expression data using realtime quantitative PCR and the $2{ }^{\Delta \Delta C T}$ method. Methods. 2001;25(4):402-8.

\section{Publisher's Note}

Springer Nature remains neutral with regard to jurisdictional claims in published maps and institutional affiliations.
Ready to submit your research? Choose BMC and benefit from:

- fast, convenient online submission

- thorough peer review by experienced researchers in your field

- rapid publication on acceptance

- support for research data, including large and complex data types

- gold Open Access which fosters wider collaboration and increased citations

- maximum visibility for your research: over $100 \mathrm{M}$ website views per year

At BMC, research is always in progress.

Learn more biomedcentral.com/submissions 